${ }^{1}$ Department of Travel, Tourism and Recreation Services, Ardeşen Vocational School, Recep Tayyip Erdoğan University, Rize, Turkey,

burhan.basaran@erdogan.edu.tr

ORCID: 0000-0001-6506-6113

Submitted: $21 / 01 / 2021$

Revised: 20/02/2021

Accepted: 28/02/2021

Online Published: 25/03/2021

Citation: Başaran, B., The past, present and future ISO 9001 quality management system standard, bmij (2021) 9 (1): 227-247, doi:

https://doi.org/10.15295/bmij.v9i1.1756

\section{The past, present and future ISO 9001 quality management system standard}

\author{
Geçmiş, bugün ve gelecekte ISO 9001 kalite yönetim sistemi standardı
}

Burhan Başaran ${ }^{1}$

\begin{abstract}
The number of studies for the future improvement of the standard ISO 9001, which is the most used standard in many areas, is limited. Within this research scope, the number of ISO 9001 certificates registered between 1993 and 2019 in some G20 countries was evaluated, and the number of certificates for 2020-2026 was estimated using the Eviews 11 program. Time series regression and exponential smoothing methods are used in estimation calculation. According to the number of certificates estimated for 2026, country rankings were determined as India $(43,947)$, Brazil $(22,744)$, Canada $(11,814)$, USA $(37,235)$, Mexico $(9,977)$, Argentina $(8,433)$, Russian Federation $(1,540)$, Australia $(5,072)$, South Africa $(4,626)$ and Saudi Arabia $(2,785)$. Changes are expected in Canada, the Russian Federation, Australia and Mexico in 2026 when the 2019 certificate number of countries' ranking is considered. A decrease in Australia and the Russian Federation's certificate number was foreseen for 2026. It is thought that the research will contribute to the interpretation of ISO 9001 performance of nine countries and to how the future trend will be shaped.
\end{abstract}

Keywords: ISO 9001, Time Series Analysis, Exponential Smoothing, Canada, USA

Jel Codes: C1, L15

Öz

Dünya genelinde kullanım alanı en fazla olan standart ISO 9001'in gelecekteki gelişimine yönelik çalışma sayısı sınırlıdır. Bu araştırmanın amacı G20 ülkelerinin 1993-2019 yılları arasında kaydedilen ISO 9001 sertifika sayıları değerlendirilmiş ve 2020-2026 yılı sertifika sayıları ise Eviews 11 programı kullanılarak tahmin edilmiştir. Tahmin hesaplamasında time series regression ve exponential smoothing yöntemlerine ait modellerden faydalanılmıştır. Araştırmada 2026 yılı için tahmin edilen sertifika sayısına göre ülke sıralaması, Hindistan (43947), Brezilya (22744), Kanada (11814), ABD (37235), Meksika (9977), Arjantin (8433), Rusya Federasyonu (1540), Avustralya (5072), Güney Afrika (4626) ve Suudi Arabistan (2785) olarak belirlenmiștir. Ülkelerin 2019 yılı sertifika sayısı sıralaması referans alındığında 2026 yılında Kanada, Rusya Federasyonu, Avustralya ve Meksika'nın pozisyonunda değişiklikler beklenmektedir. 2026 yılında Avustralya ve Rusya Federasyonu'nın sertifika sayısında azalma öngörülmüştür. Araştırmanın özellikle belirlenen dokuz ülkenin ISO 9001 performansının ve gelecekteki trendin nasıl şekilleneceğinin anlaşılmasına katkıda bulunacağı düşünülmektedir.

Anahtar Kelimeler: ISO 9001, Zaman Serileri Analizi, Üstsel Düzeltme, Kanada, ABD

JEL Kodları: C1, L15 


\section{Introduction}

In the globalisation and information age, which is experienced now, the improvements that took place during a few centuries have been experienced in a few years today. Furthermore, societies' habits have changed, and the world economy has been reshaped (Petricevic and Teece, 2019; Prabhakar and Erokhin, 2020). In parallel with the intensive economic, political and social relations between countries, the product/service produced in any region has become marketable to another customer/consumer group (Başaran, 2016). This situation has led to substantial economic competition (Ross, 2020; Rodriguez-Arnaldo and Martínez-Lorente, 2020). Therefore, organisations have to adapt to stakeholders' changing expectations and develop new flexible, competitive strategies to keep up with this future trend. Within the scope of this new competitive strategy, organisations have to systematically monitor the improvements, evaluate the data flow systematically, and use new production/marketing methods by using their resources effectively and ensuring sustainable development (Başaran, 2018; Barros et al., 2020). Management systems standards (MSSs) have been regarded as a strategic tool to conduct the whole process successfully (Priede, 2012; Simon, Yaya, Karapetrovic and Casadesús, 2014; Nunhes, Barbosa and de Oliveira, 2017; Ikram, Zhang and Sroufe, 2020a).

MSSs are published by the International Organization for Standardization (ISO). ISO was founded in 1947 and is headquartered in Geneva, Switzerland. This independent organisation comprises 165 members (4 subscribe, 39 correspondent and 122 member bodies - 792 technical committees) from different countries. This organisation's primary objective is to promote international standards according to the needs of the public and private sectors, conduct applications and inspections, eliminate the differences in standards between countries, and contribute to the development of trade. Within this scope, 23,432 international standards, including all aspects of the trade, have been published (ISO, 2020a; 2020b). The number of A and B type MSSs developed by ISO for different purposes and areas, which are still in effect, is 80 (ISO, 2020c). While some of these standards (ISO 22000, ISO 13485, ISO 16949) are developed for various sectors, they have also published ISO 9001, ISO 14001 standards applicable in all sectors. ISO 9001 Management Quality system (QMS) is the most widely used standard globally (Saraiva and Duarte, 2003; Ikram et al., 2020a).

Historical development of ISO 9001 QMS is as follows (ISO, 2020a):

- 1963 - MIL/Q/9858 standard was prepared for the defence technology in the USA.

- 1968 - AQAP standards were initiated to be used by NATO member countries.

- 1979 - England created BS 5750 standard.

- 1987 - ISO published ISO 9000 series.

- 1988 - CEN published EN 29000 standards.

- 1988 - Published as TS 6000 Quality Assurance System Standard.

- 1994 - Revised by ISO (9001:1994/9002:1994/9003:1994).

- 1996 - EN 29000 series was published as EN ISO 9000.

- 2000 - Revised by ISO and published as ISO 9001:2000.

- 2008 - Revised by ISO and published as ISO 9001:2008.

- 2015 - Revised by ISO and referred to as ISO 9001:2015.

ISO 9001:2015 Standard covers ten sections (clauses). The first three are introductory and include general topics such as scope, referenced standards, terms and descriptions. Clause 4 is about "context of the organisation", clause 5 is about "leadership", clause 6 is about "planning", clause 7 is about "support", clause 8 is about "operation", clause 9 is about "performance evaluation", and clause 10 is about "improvement" (ISO, 2015). For an enterprise to use ISO 9001 certification in its operations, it must be audited by an accredited certification body.

Official certification bodies in different countries, acting under the International Accreditation Forum (IAF), accredit companies that comply with ISO standard certification. Accredited certification bodies, on the other hand, inspect businesses that are seeking to become ISO certified. A contract is signed between the enterprise to be certified and the certification body. Within this scope, the enterprise is audited at intervals to check its compliance with the specified standards. Hence, during this process, many enterprises seek professional support to implement the standards. This situation has led to the emergence of new certification bodies. Therefore, it can be concluded that the standards correspond to 
a huge economic value and provide advantages to businesses when the stakeholders are involved in the process.

According to the ISO 2020 report, approximately 1,217,972 companies were certified by the end of 2019 . The companies (excluding those whose sector is unknown) which have received the most ISO 9001 certification are Basic metal \& fabricated metal products $(10.37 \%)$, Wholesale \& retail trade, repairs of motor vehicles, motorcycles \& personal \& household goods $(7.75 \%)$, Electrical and optical equipment $(7.46 \%)$, Construction (6.85\%) and Machinery, and equipment (6.25\%). The top five countries with the highest ISO 9001 certification are China (23.13\%), Italy (11.30\%), Japan (6.77\%), Germany $(5.91 \%)$ and Spain $(4.87 \%)$ (ISO, 2020d).

Table 1: Total Numbers of ISO 9001 Certificate 2000-2019 (ISO, 2020d)

\begin{tabular}{|c|c|c|c|c|c|}
\hline Year & 2000 & 2001 & 2002 & 2003 & 2004 \\
\hline ISO 9001 & 407,674 & 510,349 & 561,766 & 497,919 & 660,132 \\
\hline Change (\%) & - & 25.19 & 10.07 & -11.37 & 32.58 \\
\hline Year & 2005 & 2006 & 2007 & 2008 & 2009 \\
\hline ISO 9001 & 773,843 & 896,905 & 951,486 & 980322 & $1,063,751$ \\
\hline Change (\%) & 17.23 & 15.90 & 6.09 & 3.03 & 8.51 \\
\hline Year & 2010 & 2011 & 2012 & 2013 & 2014 \\
\hline ISO 9001 & $1,076,525$ & $1,009,845$ & $1,017,279$ & $1,022,877$ & $1,036,321$ \\
\hline Change (\%) & 1.20 & -6.19 & 0.74 & 0.55 & 1.31 \\
\hline Year & 2015 & 2016 & 2017 & 2018 & 2019 \\
\hline ISO 9001 & $1,034,180$ & $1,105,937$ & $1,058,504$ & $1,180,965$ & $1,217,972$ \\
\hline Change (\%) & -0.21 & 6.94 & -4.29 & 11.57 & 3.13 \\
\hline
\end{tabular}

When the total number of ISO 9001 certificates between 2000 and 2019 is examined (Table 1), it is deduced that although a decrease was observed in some years, the number of certificates broadly increased compared to the previous years; however, there was a decrease in the rate of increase. For example, a decrease of $11.37 \%, 6.19 \%$, and $4.19 \%$ was observed in the transition from 2002 to 2003,2010 to 2011, and 2016 to 2017, respectively. The increase rates in 2018 (11.57\%) and 2019 (3.13\%) strengthen the idea that ISO 9001 is still perceived as an essential strategic tool for companies.

\section{Literature review}

\section{The diffusion and future of ISO 9001}

Some researchers (Saraiva and Duarte, 2003; Franceschini, Galetto and Gianni, 2004; Viadiu, Fa and Saizarbitoria, 2006; Sampaio, Saraiva and Rodrigues, 2011; Salgado, Beijo, Sampaio, Mello and Saraiva, 2016; Cabecinhas, Domingues, Sampaio and Arezes, 2019; Ikram et al., 2020a) pointed out the lack of research in the literature examining the diffusion and future of ISO 9001. Within this scope, these researchers conducted studies using various mathematical models. Saraiva and Duarte (2003) developed a regression model regarding country population, gross national income, and the number of certificates in the past two years (2000 and 2001). They allowed the calculation of ISO 9001 certification estimates to explain the ISO 9001 certification diffusion and predict how it will evolve around the world in the future (2002-2006). The abovementioned authors stated that economic developments positively affected the number of ISO 9001 certificates.

Franceschini et al. (2004) examined the diffusion and future of ISO 9001 certification in some European countries. The logistic model is used in this research, depending on a close analogy between certificate issuance and bio-population growth. This model provides a forecast of the required time to reach a saturation level for certificates and the future of new certificates. The researchers stated that following a rapid growth period when the certified companies reach a specific limit, the certificate loses its positive connotation and becomes less attractive. As a result of this situation, a significant decrease is expected in the number of enterprises potentially interested in certification. Franceschini et al. (2004) raised the following questions about the future of ISO 9001 as many countries have reached the saturation level: Will the certification market continue to grow? Will the certified enterprises continue to be interested in the issue? Can new certification approaches be foreseen? If yes, what are the new aspects of quality?

Sampaio et al. (2011) used the exponential smoothing forecasting model (Brown Model) to characterise the development of countries' ISO 9001 certification and to estimate the number of certificates for 20072010 by considering the ISO 9001 data belonging to the period of 1993-2006. They classified the countries according to ISO 9000 development models, and models were developed for each cluster. However, it was stated that the developed model is not a unique forecasting model that can be implemented in all countries. The authors raised the following questions about ISO 9001 certification: Will the certification 
market continue to grow? Are there other variables that could influence the ISO 9000 countries' diffusion? Are there other statistical models that could better characterise the ISO 9000 evolution?

Salgado et al. (2016) divided 18 countries in the American continent into six clusters according to various economic indicators, and regression models were developed to characterise these countries' certification evolution. A positive relationship was found between the number of issued certificates in each country per 1000 inhabitants and economic development indicators (Gross National Income Per Capita).

Cabecinhas et al. (2019) examined the diffusion and forecasting models of Portugal integrated management systems (IMS) covering ISO 9001, ISO 14001 and OHSAS 18001 standards using logistic curve models. They reported that the certificate diffusion showed S-shaped behaviour, and reaching a certain saturation level might differ in each country.

Ikram et al. (2020a) analysed the ISO 9001 certification diffusion and future (2018-2026) in China, Italy, Germany, Japan, United Kingdom and India by using a novel grey forecasting approach (Even Grey Model, Discrete Grey Model, Nonhomogeneous Discrete Grey Model). They reported that China and India have higher ISO 9001 certification growth stages compared to other countries. These two countries are followed by Italy, Germany, Japan and the U.K., respectively. At the end of their studies, they focused on the question, "can future research investigate the relationship between certification bodies and the growth of certification?"

It is possible to say that a reasonable number of researchers focused on the ISO 9001 standard, which is one of the most common standards all over the world. Despite such an extensive study and rich content, the number of studies focusing on future trends of ISO 9001 is quite limited. Many researchers also emphasised that more factual and statistically focused studies should be carried out in the field (Sampaio et al., 2011; Salgado et al., 2016; Ikram et al., 2020a). This study's primary objective is to assess the ISO 9001 data of some G20 countries for the years 1993-2019 and to predict the certificate numbers in 2020-2026 using time series analysis.

\section{Research methodology}

The ISO 9001 certificate numbers published by ISO for the years 1993-2019 constitute this research material. Two criteria were taken into account in-country selection: (i) being a G20 country and (ii) being a country where a few or no studies have been conducted. Within this context, ten countries were determined. These are India, Mexico, Canada, the USA, Argentina, Australia, South Africa, Saudi Arabia, Brazil, and the Russian Federation.

In this research, instead of using a single model, different time series analysis methods that best explain the ISO 9001 developments of the countries were used to predict the future number of ISO 9001 certifications of countries. Time series analysis is a method that enables the statistical analysis of data observed at regular intervals over time, and it makes a reliable prediction of the data that can be collected in future periods. Forecasting of future events provides critical inputs for the planning and decision-making process in many areas such as managing operations such as business, production, provisions and staff, predicting the market size of the sector, economy, finance and risk management of private and public institutions, and the change in demographic characteristics. (Montgomery et al., 2015). Mert and Çağlar (2019) suggested two different time series analysis methods, namely time series regression and exponential smoothing, to be used in prediction studies for short series where the number of observations is low $(<30)$. The methods used in this study based on the reference are explained below.

\section{Time series regression}

This method is used for modelling the trend in deterministic structure in time series. Because of the annual series and no seasonal effect on the annual series, non-seasonal time series regression models are used in this study. Ten different models given below are used in modelling the series. In the models, the variable $t$, which takes consecutive values starting from 0 and is an indicator of the linear trend, is used as the independent variable(s). The future predictions are made by curve fitting to the deterministic trend structures common in these models (Mert and Çağlar, 2019).

\section{Used regression models}

1. The simple linear regression model

$y_{t}=a+b t$

2. First difference linear regression model

$\Delta y_{t}=a+b t$ 
3. Exponential regression model

$\operatorname{Ln}\left(y_{t}\right)=a+b t$

4. Quadratic regression model

$y_{t}=a+b t+c t^{2}$

5. Logistic regression model

$\operatorname{Ln}\left(\frac{L}{y_{t}}-1\right)=a+b t$

The L value is an arbitrary value chosen to be a more significant number than the most extensive observation series.

6. Cubic regression model

$y_{t}=a+b t+c t^{2}+d t^{3}$

7. Logarithmic regression model

$y_{t}=a+b \cdot \operatorname{Ln}(t)$

8. Power regression model

$\operatorname{Ln}\left(y_{t}\right)=a+b \cdot \operatorname{Ln}(t)$

9. S-shaped regression model

$\operatorname{Ln}\left(y_{t}\right)=a+b\left(\frac{1}{t}\right)$

The $t$ variable is started from 1 as the logarithm function is undefined at zero in 7,8 and 9 regression models $(1,2,3 \ldots)$.

10. Inverse regression model

$y_{t}=a+b\left(\frac{1}{t}\right)$

As $1 / \mathrm{t}$ function is undefined at zero, $\mathrm{t}$ variable starts with $1(1,2,3 \ldots)$

In these equations (1-10) $y_{t}$ time-series (dependent variable), a constant-coefficient and $\mathrm{b}, \mathrm{c}$, and $\mathrm{d}$ are the relevant trend terms' coefficients.

\section{Exponential smoothing}

\section{Simple exponential smoothing}

Exponential smoothing is used when the time series's trend structure is deterministic or stochastic and even when the number of observations in the series is small. Contrary to the prediction of fixedparameter regression in time series, the logic of this method is to make a prediction focusing on the series's former values (Mert and Çağlar, 2019). The double exponential smoothing and Holt-Winters no seasonal smoothing methods were used as the series were trendy and annual in the study, and no seasonality was found.

1. Double exponential smoothing

$$
\begin{aligned}
& S_{T}=a y_{t}+(1-\alpha) S_{T-1} \quad D_{T}=a S_{T}+(1-\alpha) D_{T-1} \\
& \hat{y}_{T+k}=\left(2+\frac{\alpha k}{1-\alpha}\right) S_{T}-\left(1+\frac{\alpha k}{1-\alpha}\right) D_{T}=\left(S_{T}-D_{T}+\frac{\alpha k}{1-\alpha}\left(S_{T}-D_{T}\right) k\right)
\end{aligned}
$$

In this equation, $S_{T}$ indicates single exponential smoothing, $D_{T}$ indicates double exponential smoothing. $T$ is the last period of time series, $k$ is the forecast period, $a$ is smoothing parameter, $\hat{y}$ is the prediction, in other words, predictive value.

2. Holt-Winters no seasonal smoothing

$\hat{y}_{t+k}=a+b k$

In this equation, $b$ trend reflects the $a$ series level (intercept).

$$
\begin{aligned}
& a(t)=\alpha y_{t}+(1-\alpha)(a(t-1)+b(t-1)) \\
& b(t)=\beta(a(t)-a(t-1))+1-\beta b(t-1)
\end{aligned}
$$


In 14 and 15 numbered equations, $(0<a, \beta<1)$ are called damping factors. Based on these equations, the predictions in Holt-Winters no seasonal smoothing method are as in equation 16.

$$
\hat{y}_{T+k}=a(T)+b(T) k
$$

In this equation (16), $T$ is the last period of time series, $k$ is the prediction period, $\hat{y}$ is the prediction, in other words predictive value.

\section{ETS exponential smoothing}

In ETS (Error, Trend, Seasonal) smoothing, the linear acceleration of the predictive values (especially if the prediction period is long) obtained from the solely additive or purely multiplicative trend component of the three essential components of the time series, error, trend and seasonal is not factual (Mert and Çağlar, 2019).

$$
y_{t}=T_{t} \times S_{t}+E_{t}, y_{t}=\left(T_{t}+S_{t}\right)\left(1+E_{t}\right)
$$

In this equation, $y_{t}$ is the time series, $E_{t}$ is the error component, $T_{t}$ is the trend component, and $S_{t}$ is the seasonal component.

It is tried to make more factual predictions in ETS exponential smoothing method for the same prediction period described above by adding a kind of damping parameter to the additive and multiplicative trend components. Trend component appears in five different ways in time series with this approach (Mert and Çağlar, 2019). These are given below.

a. No trend (None: $\mathrm{N}): T_{h}=l$

b. Additive trend (Additive: A): $T_{h}=l+b h$

c. Dampened Additive trend (Additive Dampened: $\mathrm{AD}$ ): $T_{h}=l+b \phi_{h}$

d. Multiplicative trend (Multiplicative: M): $T_{h}=l b^{h}$

e. Dampened multiplicative trend (Multiplicative Dampened: M.D.): $T_{h}=l b^{\phi_{h}}$

In these (18-22) equations, $T$ is the trend, $b$ is the linear trend variable (growth term), $h$ is the forecast period, $l$ is the series level, and $\phi$ is the damping parameter $\left(0<\phi<1\right.$ and $\left.\phi_{h}=\sum_{s}^{h} \phi^{s}\right)$.

According to the ETS exponential smoothing method, the error component in a time series is A or M, and the seasonal component is again $\mathrm{A}, \mathrm{M}$ or $\mathrm{N}$. Therefore, the possible states of the $\mathrm{E}, \mathrm{T}$ and $\mathrm{S}$ components are as follows:

$$
\begin{aligned}
E & =\{A, M\} \\
T & =\{N, A, M, A D, M D\} \\
S & =\{N, A, M\}
\end{aligned}
$$

In this method, three possible fundamental components $(\mathrm{E}, \mathrm{T}, \mathrm{S})$ are considered, a time series can be estimated with $2 \times 5 \times 3=30$ different ETS models. The seasonal component was fixed as $S=\{N\}$ since seasonality was not observed in this study; therefore, $2.5=10$ different exponential smoothing models were used in this study.

Two different estimators were suggested for the ETS model (Mert and Çağlar 2019). These are as follows:

1. The maximum likelihood (ML) estimator given by normal (Gaussian) likelihood function

$$
\log \mathrm{L}\left(\theta, \mathrm{x}_{0}, \sigma^{2} \mid \mathrm{y}\right)=-\frac{2}{\mathrm{~T}} \log \left(2 \pi \sigma^{2}\right)-\frac{1}{2} \sum_{t=1}^{n} \frac{e_{t}^{2}}{\sigma^{2}}
$$

The parameters $\theta$ that maximise the function Log-likelihood $(\log L)$ given in the above equation, and the initial values $\left(x_{0}\right)$ for the level, trend, and seasonal components of the series obtained using the Broyden, Fletcher, Goldfarb and Shanno (BFGS) algorithm. $e_{t}$ is error component, $n$ is no of observation, $y$ is time series, and $t$ is the time index.

\section{Mean squared error mean (Average MSE: AMSE)}

$$
A M S E=\frac{1}{T} \sum_{t=1}^{T}\left(\frac{1}{h} \sum_{k=1}^{h} e_{t+k \mid t}^{2}\right)
$$

Using Broyden, Fletcher, Goldfarb (BFGS) algorithm, estimators minimising the AMSE value are collected.

$T$ is the trend, $e_{t}$ is the error component, $t$ is the time series, and $h$ is the length of the forecast period. 
Among the estimated ten different ETS models, Akaike info criterion (AIC), Schwarz criterion (BIC) and Hannan-Quinn criterion (H.Q.) information criteria were used to define the best. The information criteria are as follows:

$$
\begin{aligned}
& A I C=-2 \log L\left(\hat{\theta}, \hat{x}_{0}\right)+2 p \\
& B I C=-2 \log L\left(\hat{\theta}, \hat{x}_{0}\right)+\log (T) p \\
& H Q=-2 \log L\left(\hat{\theta}, \hat{x}_{0}\right)+2 \log (\log T) p
\end{aligned}
$$

In these (25-27) equations, $\hat{\theta}, \hat{x}_{0}$ indicate estimators, $p$ is no of parameters, and $T$ indicates no of observations in the series.

\section{Model choice}

The suitable model for estimating the number of ISO 9001 certified countries was determined in three stages. (i) Tables were created by considering the models in which trend coefficients were significant $(p<0.01)$ in time series regression models, and AIC, BIC, H.Q. information criteria in ETS exponential smoothing models. (ii) Which of the models best fitting the series was determined according to statistics obtained from errors. Some of these main statistics are Root Mean Squared Error (RMSE), Mean Absolute Error (MAE), Mean Absolute Percent Error (MAPE) and Theil $U_{1}$ coefficient $\left(U_{1}\right)$ and Theil $U_{2}$ coefficient $\left(\mathrm{U}_{2}\right)$, (iii) These statistics are expected to be small in a good model. Therefore, the smallest value in each column is marked in bold. The model pointed out by these statistics was determined to be the best model. In case of equality, the smallest value in $U_{2}$ column is taken into account.

$$
\begin{aligned}
& R M S E=\sqrt{\frac{\sum_{t=1}^{T} e_{t}^{2}}{T}} \\
& M A E=\frac{\sum_{t=1}^{T}\left|e_{t}\right|}{T} \\
& M A P E=100 \times \frac{\sum_{t=1}^{T} \frac{\left|e_{t}\right|}{T}}{T} \\
& U 1=\frac{\sqrt{\frac{\sum_{t=1}^{T} e_{t}^{2}}{T}}}{\sqrt{\frac{\sum_{t=1}^{T} y_{t}^{2}}{T}+\sqrt{\frac{\sum_{t=1}^{T} \hat{y}_{t}^{2}}{T}}}} \\
& U 2=\frac{\sqrt{\sum_{t=1}^{T-1}\left(\frac{y_{t+1}-y_{t+1}}{y_{t}}\right)^{2}}}{\sqrt{\sum_{t=1}^{T-1}\left(\frac{y_{t+1}-y_{t}}{y_{t}}\right)^{2}}}
\end{aligned}
$$

In these (28-32) equations, $e_{t}$ is the error component, $y_{t}$ is the original time series value, $\hat{y}_{t}$ is the estimated value of time series, and $\mathrm{T}$ is the number of observation of series.

\section{Data analysis and evaluation}

Eviews 11 program was used for the time series analysis of the data. The past, today and future ISO 9001 data of countries were reflected in frequency distribution. Tables and figures were used in evaluations.

\section{Results}

The time series analysis methods described in the previous section were applied separately for each country data set. Models with significant trend coefficient and information criteria were selected, and tables were created for each country. (Table 2-11). 
Table 2: Model Selection (India)

\begin{tabular}{|c|c|c|c|c|c|}
\hline & RMSE & MAE & MAPE & $\mathrm{U}_{1}$ & $\mathbf{U}_{2}$ \\
\hline Simple linear regression & $8,210.257331$ & $6,357.586985$ & 124.275012 & 0.154428 & 1.293405 \\
\hline Exponential regression & $24,278.185113$ & $14,494.228153$ & 110.465323 & 0.366192 & 3.022816 \\
\hline Quadratic regression & $7,353.470570$ & $5,841.439974$ & 559.280667 & 0.137650 & 17.057920 \\
\hline Logistic regression & $14,258.595421$ & $9,388.907334$ & 92.189255 & 0.252388 & 2.889545 \\
\hline Cubic regression & $6,111.689245$ & $4,371.507207$ & 177.801352 & 0.113730 & 7.073086 \\
\hline Logarithmic regression & $9,114.063361$ & $7,444.641640$ & 997.120803 & 0.172412 & 18.214922 \\
\hline Power regression & $11,899.828016$ & $7,623.250347$ & $28.107715^{*}$ & 0.209586 & 0.528334 \\
\hline S-shaped regression & $11,901.413574$ & $9,908.968960$ & 75.954361 & 0.264787 & 2.143734 \\
\hline Inverse regression & $13,141.045751$ & $11,979.399261$ & $1,207.437081$ & 0.257411 & 16.615926 \\
\hline Double exponential smoothing & $5,962.539703$ & $4,263.301849$ & 362.981187 & 0.107630 & 16.277711 \\
\hline Holt-Winters no seasonal smoothing & $5,364.682051$ & $4,013.780919$ & 65.037428 & 0.096464 & 8.999876 \\
\hline ETS $(\mathrm{M}, \mathrm{A}, \mathrm{N})(\mathrm{ML})$ & $6,292.075253$ & $3,939.399588$ & 78.974535 & 0.113376 & $0.334471^{*}$ \\
\hline ETS $(\mathrm{M}, \mathrm{A}, \mathrm{N})(\mathrm{AMSE})^{* *}$ & $5,156.121093^{*}$ & $3,490.875105^{*}$ & 79.110406 & $0.094272^{*}$ & 3.439181 \\
\hline
\end{tabular}

Note: * The smallest value in each column is marked in bold. ${ }^{* *}$ The model pointed out by these statistics was determined to be the best model.

Table 3: Model Selection (Mexico)

\begin{tabular}{|l|r|r|r|r|r|}
\hline & \multicolumn{1}{|c|}{ RMSE } & \multicolumn{1}{c|}{ MAE } & \multicolumn{1}{c|}{ MAPE } & \multicolumn{1}{c|}{$\mathbf{U}_{1}$} & $\mathbf{U}_{2}$ \\
\hline Simple linear regression ${ }^{* *}$ & $\mathbf{5 4 5 . 6 3 1 2 6 8 ^ { * }}$ & $\mathbf{3 9 7 . 0 8 6 0 2 8}$ & 82.697826 & 0.061301 & 1.779907 \\
\hline Exponential regression & $2,731.383069$ & $1,636.000453$ & 88.164072 & 0.257016 & 2.924443 \\
\hline Logistic regression & $2,411.316032$ & $1,494.264564$ & 85.612510 & 0.233167 & 2.895040 \\
\hline Logarithmic regression & $1,141.289489$ & 932.841881 & 508.284736 & 0.129898 & 11.103517 \\
\hline Power regression & 999.755785 & 718.419638 & $\mathbf{2 2 . 3 9 7 3 7 7 ^ { * }}$ & 0.105691 & $\mathbf{0 . 5 6 4 9 0 7}$ \\
\hline S-shaped regression & $1,563.736382$ & $1,191.951955$ & 51.217481 & 0.201204 & 2.124139 \\
\hline Inverse regression & $1,964.335704$ & $1,708.533184$ & 625.329412 & 0.231665 & 11.732368 \\
\hline Double exponential smoothing & 552.852746 & 408.062488 & 78.192065 & 0.062713 & 1.566203 \\
\hline Holt-Winters no seasonal smoothing & 622.329685 & 470.629871 & 41.211251 & 0.069563 & 3.747638 \\
\hline ETS (A,A,N) (ML) & 545.631268 & 397.086060 & 82.697982 & $\mathbf{0 . 0 6 1 3 0 1}$ & 1.779917 \\
\hline ETS (A,A,N) (AMSE) & 546.282548 & 400.113241 & 92.054612 & 0.061367 & 2.321251 \\
\hline
\end{tabular}

Note: * The smallest value in each column is marked in bold. ${ }^{* *}$ The model pointed out by these statistics was determined to be the best model.

Table 4: Model Selection (Argentina)

\begin{tabular}{|l|r|r|r|r|r|}
\hline & \multicolumn{1}{|c|}{ RMSE } & \multicolumn{1}{c|}{ MAE } & \multicolumn{1}{c|}{ MAPE } & \multicolumn{1}{c|}{$\mathbf{U}_{\mathbf{1}}$} & $\mathbf{U}_{\mathbf{2}}$ \\
\hline Simple linear regression & $1,574.709504$ & $1,098.871282$ & 209.355733 & 0.157105 & 13.155464 \\
\hline Exponential regression & $5,168.463880$ & $3,164.935421$ & 167.792051 & 0.395262 & 5.239366 \\
\hline Quadratic regression & $1,349.487925$ & 985.422252 & 775.524528 & 0.133742 & 19.543941 \\
\hline Logistic regression & $4,518.416430$ & $2,872.883950$ & 163.295513 & 0.363318 & 5.236630 \\
\hline Logarithmic regression & $1,599.798875$ & $1,272.000334$ & $1,349.024792$ & 0.159738 & 19.383156 \\
\hline Power regression & $2,893.782123$ & $2,015.613037$ & $\mathbf{4 6 . 0 8 6 3 8 1}$ & 0.252827 & 1.166219 \\
\hline S-shaped regression & $1,955.106670$ & $1,448.999732$ & 74.035886 & 0.223413 & 4.116163 \\
\hline Inverse regression & $2,293.369039$ & $2,015.905982$ & $1,831.678840$ & 0.235697 & 34.654780 \\
\hline Double exponential smoothing & $1,407.719933$ & 913.120558 & 659.399745 & 0.134408 & 21.303977 \\
\hline Holt-Winters no seasonal smoothing & $\mathbf{1 , 2 2 1 . 5 0 1 4 9 8}$ & 745.916587 & 142.937539 & 0.115969 & 15.433829 \\
\hline ETS (M,A,N) (ML) & $1,431.120172$ & 869.821854 & 145.952497 & 0.137256 & $\mathbf{0 . 7 0 7 4 1 5}$ \\
\hline ETS (M,AD,N) (AMSE) $^{* *}$ & $\mathbf{1 , 1 8 0 . 6 3 2 0 8 6}$ & $\mathbf{6 9 0 . 4 0 1 6 3 8}$ & 160.130330 & $\mathbf{0 . 1 1 4 3 8 3 ^ { * }}$ & 6.318259 \\
\hline
\end{tabular}

Note: * The smallest value in each column is marked in bold. ${ }^{* *}$ The model pointed out by these statistics was determined to be the best model. 
Table 5: Model Selection (Australia)

\begin{tabular}{|c|c|c|c|c|c|}
\hline & RMSE & MAE & MAPE & $\mathrm{U}_{1}$ & $\mathbf{U}_{2}$ \\
\hline Quadratic regression & $5,502.310941$ & $4,358.871454$ & 44.403895 & 0.195354 & 1.536488 \\
\hline Cubic regression & $4,231.522986$ & $3,617.700210$ & 35.282887 & 0.147878 & 1.021890 \\
\hline Power regression & $6,885.929724$ & $5,180.110887$ & 43.926626 & 0.260693 & 1.392707 \\
\hline S-shaped regression & $6,336.428594$ & $4,679.163624$ & 36.285799 & 0.236933 & 1.225539 \\
\hline Inverse regression & $6,099.236950$ & $4,874.957306$ & 42.789243 & 0.218550 & 1.564512 \\
\hline Double exponential smoothing & $3,857.463458$ & 3,099.129676 & 32.041478 & 0.128228 & 1.042490 \\
\hline Holt-Winters no seasonal smoothing ${ }^{* *}$ & 3,647.880198 & $2,356.034870^{*}$ & $21.534344^{*}$ & $0.121149^{*}$ & 0.858594 \\
\hline ETS $(\mathrm{M}, \mathrm{A}, \mathrm{N})(\mathrm{ML})$ & 3,775.796498 & $2,469.555836$ & 23.015042 & 0.123176 & $0.800107^{*}$ \\
\hline ETS $(\mathrm{A}, \mathrm{N}, \mathrm{N})(\mathrm{AMSE})$ & $3,613.768179^{*}$ & $2,472.358025$ & 25.056250 & 0.123725 & 1.000000 \\
\hline
\end{tabular}

Note: * The smallest value in each column is marked in bold. ${ }^{* *}$ The model pointed out by these statistics was determined to be the best model.

Table 6: Model Selection (South Africa)

\begin{tabular}{|l|c|c|c|c|c|}
\hline & RMSE & MAE & MAPE & $\mathbf{U}_{\mathbf{1}}$ & $\mathbf{U}_{\mathbf{2}}$ \\
\hline Simple linear regression & 494.639920 & 378.047302 & 14.853510 & 0.078945 & 1.117345 \\
\hline Exponential regression & 588.493518 & 420.897447 & 15.712514 & 0.093926 & 1.217864 \\
\hline Quadratic regression & $\mathbf{4 2 6 . 9 3 4 7 7 9 *}$ & 319.787034 & 10.808344 & $\mathbf{0 . 0 6 8 0 3 1}^{*}$ & 0.838057 \\
\hline Logistic regression & 583.999096 & 418.550054 & 15.649297 & 0.093235 & 1.212167 \\
\hline Logarithmic regression & 435.918132 & 350.520505 & 12.872321 & 0.069476 & $\mathbf{0 . 8 2 6 5 2 3 ^ { * }}$ \\
\hline Power regression** & 435.361378 & $\mathbf{3 1 5 . 8 0 7 1 5 2}$ & $\mathbf{1 0 . 2 2 2 3 2 5}$ & 0.069536 & $\mathbf{0 . 8 3 6 6 5 5 ^ { * * * }}$ \\
\hline S-shaped regression & 568.754348 & 468.792823 & 17.701594 & 0.092560 & 1.249887 \\
\hline Inverse regression & 644.266153 & 524.771919 & 22.810015 & 0.103279 & 1.585900 \\
\hline Double exponential smoothing & 522.296836 & 413.363957 & 14.893713 & 0.081510 & 1.006690 \\
\hline Holt-Winters no seasonal smoothing & 482.579197 & 331.980057 & 11.096769 & 0.075387 & 0.868028 \\
\hline ETS (M,A,N) (ML) & 492.678140 & 337.016328 & 11.343084 & 0.076673 & 0.864034 \\
\hline ETS (A,MD,N) (AMSE) & 432.958525 & 323.962793 & 11.271293 & 0.069087 & 0.859946 \\
\hline
\end{tabular}

Note: * The smallest value in each column is marked in bold. ${ }^{* *}$ The model pointed out by these statistics was determined to be the best model. ${ }^{* * *}$ In case of equality, the smallest value in $\mathrm{U}_{2}$ column is taken into account.

Table 7: Model Selection (Russian Federation)

\begin{tabular}{|l|r|r|r|r|r|}
\hline & \multicolumn{1}{|c|}{ RMSE } & \multicolumn{1}{c|}{ MAE } & \multicolumn{1}{c|}{ MAPE } & \multicolumn{1}{c|}{$\mathbf{U}_{\mathbf{1}}$} & $\mathbf{U}_{\mathbf{2}}$ \\
\hline Simple linear regression & $13,879.6122$ & $7,615.8906$ & $2,298.25$ & 0.510198 & 73.83960 \\
\hline Exponential regression & $19,445.9223$ & $10,658.5034$ & 249.2976 & 0.609141 & 4.218894 \\
\hline Quadratic regression & $12,843.6929$ & $7,819.4601$ & $11,202.72$ & 0.450703 & 240.5929 \\
\hline Logistic regression & $17,935.8077$ & $9,747.1166$ & 231.7222 & 0.601001 & 4.005821 \\
\hline Cubic regression & $11,770.4144$ & $7,602.7137$ & $6,968.56$ & 0.397566 & 186.8549 \\
\hline Logarithmic regression & $13,691.4737$ & $7,880.1143$ & $6,397.22$ & 0.498617 & 74.16608 \\
\hline Power regression & $15,364.7047$ & $7,488.2051$ & 102.0744 & 0.586598 & 1.603603 \\
\hline S-shaped regression & $15,178.5307$ & $6,597.0590$ & 220.7226 & 0.730294 & 7.805533 \\
\hline Double exponential smoothing & $13,697.1690$ & $6,671.4374$ & $1,134.79$ & 0.391425 & 13.26831 \\
\hline Holt-Winters no seasonal smoothing & $12,085.940264$ & $\mathbf{4 , 6 5 2 . 2 6 4 9 5 7 *}$ & 405.413711 & $\mathbf{0 . 3 5 0 0 9 1}$ & 20.113076 \\
\hline ETS (M,MD,N) (ML tahminci) & $18,204.635415$ & $7,316.469306$ & 74.790260 & 0.411068 & 0.867436 \\
\hline ETS (M,MD,N) (AMSE)** & $\mathbf{1 1 , 3 2 0 . 5 3 2 4 1 9 *}^{*}$ & $5,046.131283$ & $\mathbf{5 7 . 6 9 5 6 2 7 ^ { * }}$ & 0.382452 & $\mathbf{1 . 1 3 8 1 2 9}^{*}$ \\
\hline
\end{tabular}

Note: * The smallest value in each column is marked in bold. ${ }^{* *}$ The model pointed out by these statistics was determined to be the best model. 
Table 8. Model Selection (Saudi Arabia)

\begin{tabular}{|c|c|c|c|c|c|}
\hline & RMSE & MAE & MAPE & $\mathbf{U}_{1}$ & $\mathbf{U}_{2}$ \\
\hline Simple linear regression & 390.771164 & 310.898521 & 184.742072 & 0.139351 & 6.597712 \\
\hline Exponential regression & 739.265880 & 425.101657 & 64.881746 & 0.233644 & 1.888441 \\
\hline Logistic regression & 716.808124 & 416.446544 & 64.306099 & 0.227977 & 1.876302 \\
\hline Cubic regression & 350.281082 & 274.833154 & 56.271690 & 0.124430 & 1.772013 \\
\hline Logarithmic regression & 575.401671 & 481.766362 & 486.713080 & 0.210174 & 11.979862 \\
\hline Power regression & 374.940546 & 260.321811 & 29.571145 & 0.136602 & 0.682270 \\
\hline S-shaped regression & 741.993721 & 530.186537 & 63.074047 & 0.320838 & 1.786116 \\
\hline Inverse regression & 789.993378 & 706.214901 & 511.836425 & 0.301464 & 8.454852 \\
\hline Double exponential smoothing & 286.481506 & 188.000204 & 34.711627 & 0.098569 & 0.879792 \\
\hline Holt-Winters no seasonal smoothing & 259.013535 & 186.490028 & $23.575692^{*}$ & 0.091379 & 1.051351 \\
\hline ETS $(\mathrm{M}, \mathrm{A}, \mathrm{N})(\mathrm{ML})$ & 276.242343 & 206.789817 & 92.924468 & 0.097548 & $0.402694^{*}$ \\
\hline ETS $(\mathbf{M}, \mathrm{A}, \mathbf{N})(\mathrm{AMSE})^{* *}$ & $257.428082^{*}$ & $184.475776^{*}$ & 45.300673 & $0.090117^{*}$ & 1.837950 \\
\hline
\end{tabular}

Note: * The smallest value in each column is marked in bold. ${ }^{* *}$ The model pointed out by these statistics was determined to be the best model.

Table 9: Model Selection (Brazil)

\begin{tabular}{|l|c|c|r|r|c|}
\hline & RMSE & MAE & MAPE & $\mathbf{U}_{1}$ & $\mathbf{U}_{\mathbf{2}}$ \\
\hline Simple linear regression & $4,454.567180$ & $3,115.676028$ & 43.639960 & 0.157720 & 1.515898 \\
\hline Exponential regression & $9,519.761486$ & $6,303.855113$ & 84.457976 & 0.296026 & 2.446237 \\
\hline Quadratic regression & $4,205.347057$ & $3,126.374097$ & 150.906071 & 0.148485 & 4.724554 \\
\hline Logistic regression & $7,634.036057$ & $5,329.361321$ & 78.005685 & 0.251010 & 2.371653 \\
\hline Cubic regression & $3,596.504601$ & $2,725.712888$ & 46.215330 & 0.126228 & 1.544380 \\
\hline Logarithmic regression & $4,949.991029$ & $3,819.458851$ & 330.650737 & 0.176324 & 6.625916 \\
\hline Power regression & $5,307.209763$ & $3,713.969927$ & 29.857769 & 0.180823 & 0.654134 \\
\hline S-shaped regression & $6,020.194697$ & $4,265.635059$ & 53.355837 & 0.242710 & 1.889462 \\
\hline Inverse regression & $6,842.301731$ & $5,912.033111$ & 445.677170 & 0.251310 & 8.885501 \\
\hline Double exponential smoothing & $3,996.423402$ & $2,767.835791$ & 31.003695 & 0.135506 & 1.226889 \\
\hline Holt-Winters no seasonal smoothing** & $\mathbf{3 , 4 9 3 . 0 8 5 4 2 0}$ & $\mathbf{2 , 3 5 0 . 6 0 6 8 3 8 ^ { * }}$ & $\mathbf{2 5 . 1 9 1 4 8 7 ^ { * }}$ & $\mathbf{0 . 1 2 0 1 4 0}$ & 1.170858 \\
\hline ETS (M,A,N) (ML) & $3,589.480076$ & $2,461.708262$ & 73.648529 & 0.124213 & $\mathbf{0 . 5 0 4 8 1 7 ^ { * }}$ \\
\hline ETS (M,A,N) (AMSE) & $3,581.450824$ & $\mathbf{2 , 4 1 5 . 1 8 8 4 2 3}$ & 40.193498 & 0.123324 & 1.000122 \\
\hline
\end{tabular}

Note: * The smallest value in each column is marked in bold. ${ }^{* *}$ The model pointed out by these statistics was determined to be the best model. 
Table 10: Model Selection (Canada)

\begin{tabular}{|l|c|c|c|c|c|}
\hline & RMSE & MAE & MAPE & $\mathbf{U}_{\mathbf{1}}$ & $\mathbf{U}_{\mathbf{2}}$ \\
\hline Exponential regression & $3,838.084525$ & $3,175.770721$ & 77.074616 & 0.262510 & 3.003802 \\
\hline Quadratic regression & $1,862.350434$ & $1,608.069369$ & 37.549762 & 0.117235 & 1.946660 \\
\hline Logistic regression & $3,775.845749$ & $3,126.958130$ & 77.959088 & 0.257864 & 3.103647 \\
\hline Cubic regression $^{* *}$ & $\mathbf{1 , 3 5 1 . 3 9 9 2 7 2}$ & $\mathbf{1 , 2 0 4 . 1 1 9 9 7 2}$ & 32.834497 & $\mathbf{0 . 0 8 4 5 1 6}$ & 1.121169 \\
\hline Logarithmic regression & $\mathbf{2 , 9 6 4 . 5 6 2 7 7 1}$ & $\mathbf{2 , 5 1 7 . 4 1 0 8 7 3}$ & 71.614037 & 0.190789 & 3.567343 \\
\hline Power regression & $3,783.685777$ & $3,142.396616$ & 53.088232 & 0.246479 & 1.646530 \\
\hline S-shaped regression & $2,809.252800$ & $2,252.602641$ & 36.805390 & 0.184647 & 1.476810 \\
\hline Inverse regression & $2,643.915783$ & $2,248.186889$ & 60.080968 & 0.168859 & 3.295687 \\
\hline Double exponential smoothing & $1,904.321819$ & $1,395.715048$ & 29.200690 & 0.115288 & 0.984243 \\
\hline Holt-Winters no seasonal smoothing & $1,869.408713$ & $1,321.290620$ & $\mathbf{1 9 . 9 5 3 9 5 2}$ & 0.112765 & $\mathbf{0 . 8 3 1 6 2 0}$ \\
\hline ETS (A,N,N) (ML) & $1,880.977585$ & $1,414.695103$ & 21.559550 & 0.117323 & 1.018756 \\
\hline ETS (A,N,N) (AMSE) & $1,885.654071$ & $1,430.671089$ & 24.335491 & 0.117489 & 1.003494 \\
\hline
\end{tabular}

Note: * The smallest value in each column is marked in bold. ${ }^{*}$ The model pointed out by these statistics was determined to be the best model.

Table 11. Model Selection (USA)

\begin{tabular}{|l|r|r|r|r|c|}
\hline & \multicolumn{1}{|c|}{ RMSE } & MAE & MAPE & U $_{1}$ & $\mathbf{U}_{2}$ \\
\hline Simple linear regression & $10,095.189460$ & $8,442.168619$ & 79.118690 & 0.176545 & 5.059039 \\
\hline Exponential regression & $12,259.504175$ & $10,283.557021$ & 62.266516 & 0.220003 & 2.942007 \\
\hline Quadratic regression & $5,552.190003$ & $4,814.805427$ & 24.982055 & 0.095012 & 1.440549 \\
\hline Logistic regression & $11,306.269847$ & $9,518.243008$ & 63.687902 & 0.202652 & 3.295501 \\
\hline Cubic regression** & $\mathbf{4 , 4 3 0 . 8 3 3 0 4 3 ^ { * }}$ & $\mathbf{3 , 7 6 3 . 7 0 9 2 2 5 *}$ & 22.983197 & $\mathbf{0 . 0 7 5 5 7 4}$ & 0.655615 \\
\hline Logarithmic regression & $8,425.408707$ & $7,261.474420$ & 45.060702 & 0.145933 & 2.882805 \\
\hline Power regression & $11,393.332521$ & $9,521.651982$ & 40.315342 & 0.196408 & 1.507184 \\
\hline S-shaped regression & $7,594.564103$ & $6,234.523383$ & 25.866939 & 0.133174 & 1.302109 \\
\hline Inverse regression & $7,600.579947$ & $6,552.559948$ & 47.958819 & 0.131118 & 3.105848 \\
\hline Double exponential smoothing & $5,203.476190$ & $4,215.541846$ & 20.078074 & 0.086753 & 0.634878 \\
\hline Holt-Winters no seasonal smoothing** & $4,944.353107$ & $3,862.640058$ & $\mathbf{1 4 . 1 9 1 4 7 3}$ & 0.081896 & 0.609854 \\
\hline ETS (M,A,N) (ML) & $4,959.529677$ & $3,849.571992$ & 14.275322 & 0.081893 & 0.609912 \\
\hline ETS (M,A,N) (AMSE) & $4,944.719573$ & $3,865.361864$ & 14.224758 & 0.081884 & $\mathbf{0 . 5 8 2 4 4 9 9 ^ { * }}$ \\
\hline
\end{tabular}

Note: * The smallest value in each column is marked in bold. ${ }^{*}$ The model pointed out by these statistics was determined to be the best model.

Within the light of the data given in Table 2-11, ETS (M, A, N) (AMSE) for India and Saudi Arabia, simple linear regression for Mexico, ETS (M, A.D., N) (AMSE) for Argentina, cubic regression for Canada and USA, ETS (M, MD, N) (AMSE) for Russian Federation, Holt-Winters no seasonal smoothing for Australia and Brazil and power regression for South Africa were selected as the most suitable models. The number of ISO 9001 certificates was estimated by applying the selected model to that country's data set. The past and current ISO 9001 certificate numbers of countries for the years 19932019 and estimates for 2020-2026 are indicated in Table 12 and Figure 1-10 on the country basis. 
Table 12: Past, Present and Future ISO 9001 Certificate Numbers of Countries

\begin{tabular}{|c|c|c|c|c|c|c|c|c|c|c|}
\hline Years & India & Mexico & Argentina & Australia & $\begin{array}{l}\text { South } \\
\text { Africa }\end{array}$ & $\begin{array}{l}\text { Saudi } \\
\text { Arabia }\end{array}$ & Brazil & $\begin{array}{c}\text { Russian } \\
\text { Federation }\end{array}$ & Canada & USA \\
\hline 1993 & 73 & 24 & 9 & 2,695 & 1,007 & 10 & 113 & 5 & 530 & 2,059 \\
\hline 1994 & 328 & 85 & 23 & 3,710 & 1,161 & 30 & 384 & 8 & 870 & 3,960 \\
\hline 1995 & 1,023 & 215 & 86 & 8,834 & 1,454 & 98 & 923 & 22 & 1,397 & 8,762 \\
\hline 1996 & 1,665 & 412 & 302 & 7,252 & 1,882 & 159 & 1,198 & 56 & 3,955 & 12,613 \\
\hline 1997 & 2,865 & 711 & 397 & 10,547 & 1,915 & 211 & 2,068 & 95 & 5,852 & 18,581 \\
\hline 1998 & 3,344 & 978 & 807 & 14,170 & 2,166 & 280 & 3,712 & 132 & 7,585 & 24,987 \\
\hline 1999 & 5,200 & 1,556 & 1,388 & 22,833 & 3,316 & 324 & 6,257 & 541 & 10,556 & 33,054 \\
\hline 2000 & 5,682 & 1,843 & 2,056 & 24,772 & 3,454 & 610 & 6,719 & 1,134 & 11,435 & 35,018 \\
\hline 2001 & 5,554 & 2,233 & 2,324 & 26,750 & 2,263 & 705 & 9,489 & 1,517 & 11,635 & 37,026 \\
\hline 2002 & 8,110 & 2,508 & 2,260 & 27,135 & 2,625 & 558 & 7,900 & 1,710 & 12,371 & 38,927 \\
\hline 2003 & 8,367 & 1,437 & 1,790 & 19,975 & 2,356 & 247 & 4,012 & 962 & 8,454 & 30,294 \\
\hline 2004 & 12,558 & 3,391 & 4,149 & 17,365 & 2,486 & 394 & 6,120 & 3,816 & 9,286 & 37,285 \\
\hline 2005 & 24,660 & 2,890 & 5,556 & 16,992 & 3,119 & 642 & 8,533 & 4,883 & 12,503 & 44,270 \\
\hline 2006 & 40,967 & 4,636 & 7,934 & 17,440 & 3,259 & 710 & 9,014 & 6,398 & 11,917 & 44,883 \\
\hline 2007 & 46,091 & 3,946 & 8,808 & 7,401 & 3,283 & 645 & 15,384 & 11,527 & 7,462 & 36,192 \\
\hline 2008 & 37,958 & 4,990 & 8,812 & 8,773 & 3,792 & 876 & 12,057 & 16,051 & 10,506 & 32,400 \\
\hline 2009 & 37,493 & 5,020 & 4,428 & 9,143 & 3,545 & 1,150 & 13,452 & 53,152 & 7,992 & 28,935 \\
\hline 2010 & 33,932 & 4,259 & 5,093 & 8,784 & 3,326 & 1,339 & 26,663 & 62,225 & 7,272 & 25,101 \\
\hline 2011 & 29,574 & 4,611 & 4,753 & 9,659 & 3,409 & 1,644 & 28,325 & 13,308 & 7,108 & 25,811 \\
\hline 2012 & 28,600 & 5,502 & 6,605 & 9,185 & 3,917 & 2,189 & 25,791 & 12,488 & 6,907 & 26,177 \\
\hline 2013 & 40,848 & 5,364 & 6,634 & 13,123 & 3,565 & 2,328 & 22,128 & 11,764 & 8,346 & 34,869 \\
\hline 2014 & 40,481 & 7,338 & 6,741 & 13,945 & 3,766 & 2,759 & 18,196 & 11,213 & 5,996 & 28,125 \\
\hline 2015 & 36,305 & 7,418 & 7,112 & 13,636 & 4,346 & 3,024 & 17,529 & 9,084 & 6,417 & 33,103 \\
\hline 2016 & 37,052 & 7,027 & 7,059 & 12,765 & 4,761 & 2,353 & 20,908 & 5,083 & 6,751 & 30,474 \\
\hline 2017 & 36,053 & 7,184 & 6,423 & 12,163 & 4,255 & 2,233 & 17,165 & 3,490 & 5,947 & 25,087 \\
\hline 2018 & 31,795 & 6,535 & 6,198 & 6,672 & 3,257 & 1,796 & 16,351 & 4,497 & 4,894 & 21,848 \\
\hline 2019 & 34,397 & 7,741 & 6,611 & 7,184 & 3,464 & 2,206 & 17,952 & 4,134 & 4,557 & 20,956 \\
\hline 2020 & 35,761 & 8,093 & 6,871 & 6,882 & 4,250 & 2,288 & 18,636 & 3,507 & 5,593 & 24,270 \\
\hline 2021 & 37,125 & 8,407 & 7,131 & 6,580 & 4,316 & 2,371 & 19,321 & 3,161 & 6,025 & 24,984 \\
\hline 2022 & 38,489 & 8,721 & 7,392 & 6,278 & 4,380 & 2,454 & 20,006 & 2,541 & 6,671 & 26,204 \\
\hline 2023 & 39,854 & 9,035 & 7,652 & 5,977 & 4,443 & 2,537 & 20,690 & 2,127 & 7,552 & 27,985 \\
\hline 2024 & 41,218 & 9,349 & 7,912 & 5,675 & 4,505 & 2,620 & 21,375 & 1,834 & 8,689 & 30,381 \\
\hline 2025 & 42,582 & 9,663 & 8,173 & 5,373 & 4,566 & 2,703 & 22,060 & 1,632 & 10,102 & 33,446 \\
\hline 2026 & 43,947 & 9,977 & 8,433 & 5,072 & 4,626 & 2,785 & 22,744 & 1,540 & 11,814 & 37,235 \\
\hline
\end{tabular}

Note: * The \% values for the year 2020-2026 was calculated based on 2019 data.

When the numbers of ISO 9001 issued to India are examined (Table 12), a significant increase is observed in 2002, 2004, 2005 and 2006 compared to the previous years. The highest number of certificates was reached in 2007. In the five years following 2007, there was a continuous decrease. However, the number of certificates increased again in the transition from 2012 to 2013. In the period from 2013 to 2018, the number of certificates tended to decrease once again. When the year 2000 was taken as reference (Figure 1 ), there were first upward and then downward movements in the number of certificates, especially in 6-7 year periods. The increase in the transition from 2018 to 2019 points out that India entered a rising period again. It is predicted that the number of certificates in India will increase continuously. Accordingly, the estimated number of certificates in 2026 was estimated as 43,947, with an increase of $27.76 \%$ compared to 2019. The estimated result is below the highest value $(46,091)$ India reached in 2007.

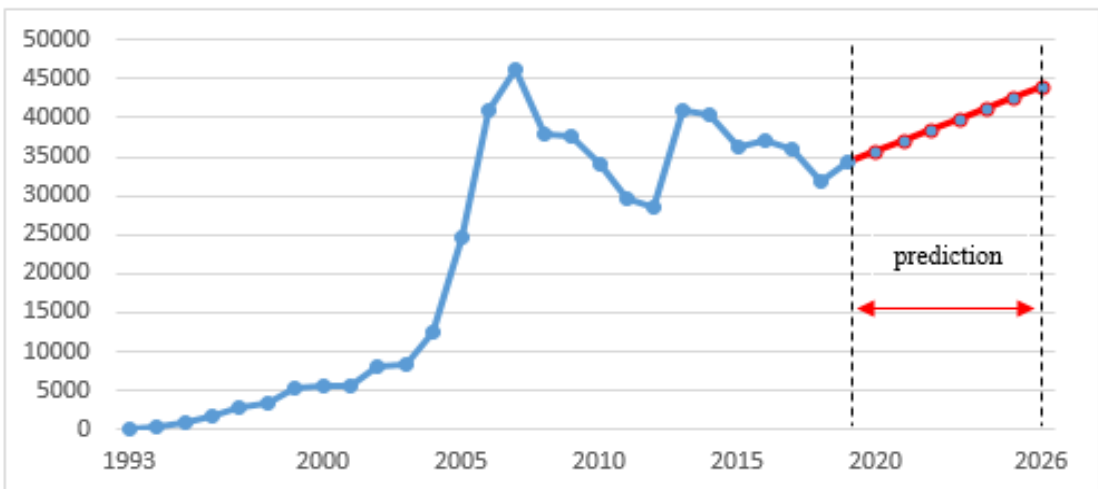

Figure 1. Estimated ISO 9001 Certification Number (India)

In light of the data in Table 12, it is seen that the number of ISO 9001 certificates in Mexico continuously increased from 1993 to 2002 . When Figure 2 is analysed, it is seen that there has been a periodic decrease 
and increase in the number of certificates since 2002. What is striking here is that the increase observed after the decrease exceeds the increase rate in the previous rising year. The years 2002, 2004, 2006, 2009, 2012 and 2014 can be regarded as examples. The highest number of ISO 9001 certificates $(7,741)$ was reached in 2019. This study predicted that there would be a continuous increase in the number of certificates in 2020-2026, and the number of certificates is estimated to be 9,977 in 2026.

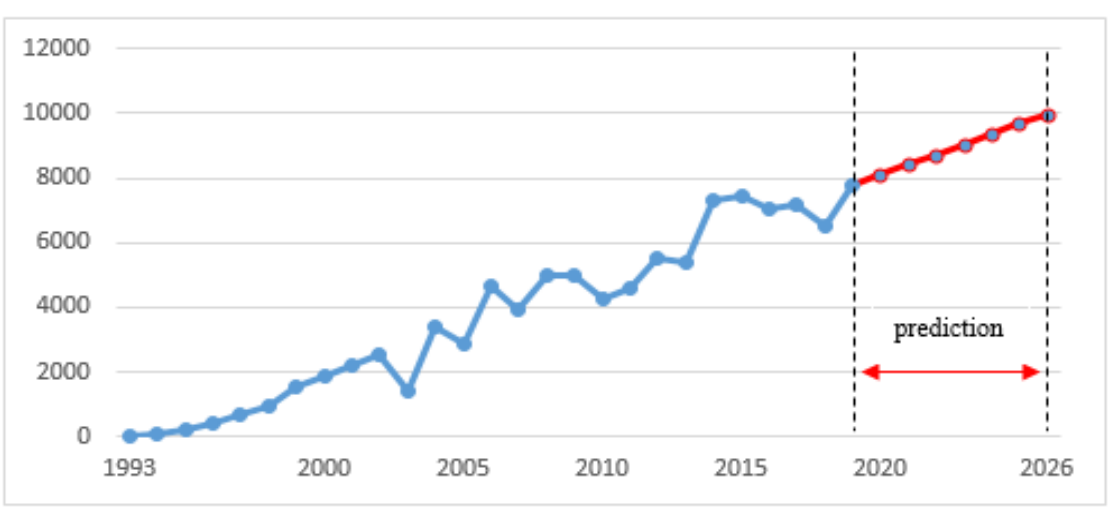

Figure 2. Estimated ISO 9001 Certification Number (Mexico)

When the data on Argentina are examined (Table 12, Figure 3), it is seen that the number of ISO 9001 certificates continuously increased in the nine years from 1993 to 2001. The number of certificates reached the highest level in 2004-2008. After 2008, the number of certificates followed a fluctuating course, and the number of certificates in 2008 could not be reached again in the later years, including 2019. This research predicted that the number of certificates for Argentina would increase continuously between 2020 and2026. Furthermore, it is estimated that the number of certificates in 2026 will reach 8,433 , with an increase of $27.56 \%$ compared to 2019 . This result is lower than the 2008 value $(8,812)$ when the highest number of certificates is observed.

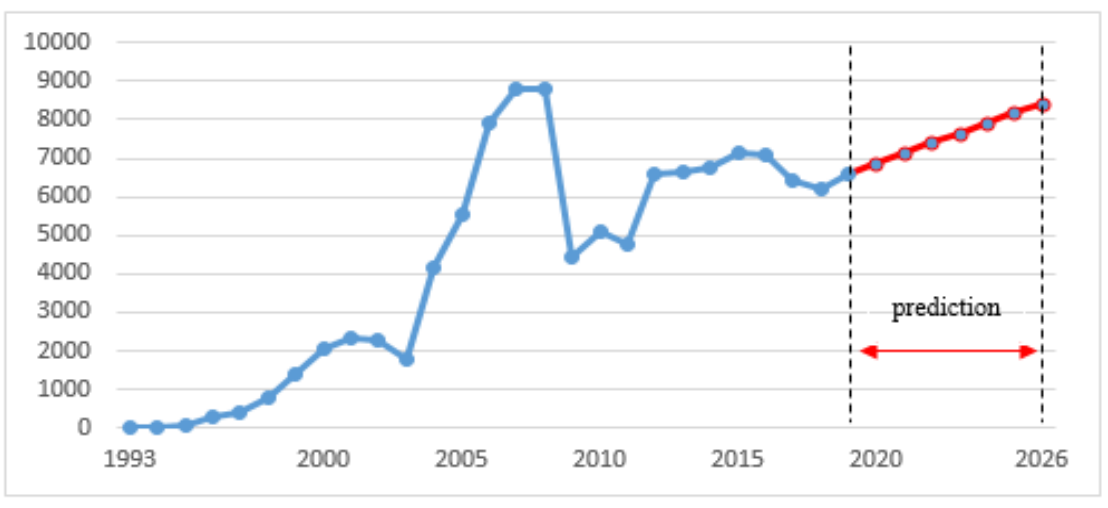

Figure 3. Estimated ISO 9001 Certification Number (Argentina)

When the data on Australia are examined (Table 12), it is understood that the number of ISO 9001 certificates continuously increased in the period from 1993 to 2002 (except 1996). The number of certificates reached in 2002 was approximately nine times the value in 1993. The highest number of certificates $(27,135)$ between 1993 and 2019 was reached in 2002. In the period from 2002 to 2012, there was a severe decrease in the number of certificates. The decrease of $57.5 \%$ from 2006 to 2007 is also remarkable. Partial increases were recorded in the same period. In 2013, another jump (approximately $43.0 \%$ increase) was experienced in the number of certificates. Especially in the transition from 2017 $(12,163)$ to $2018(6,672)$, a dramatic decrease in the number of certificates was observed (Figure 4). The number of certificates in 2018 is the third lowest value in the 26 years (1993-2018) after 1993 and 1994. This research predicted that Australia's future ISO 9001 certification number would be in a continuous decrease trend. The number of certificates in 2026 is estimated to be 5,072, with a decrease of $29.4 \%$ compared to 2019. 


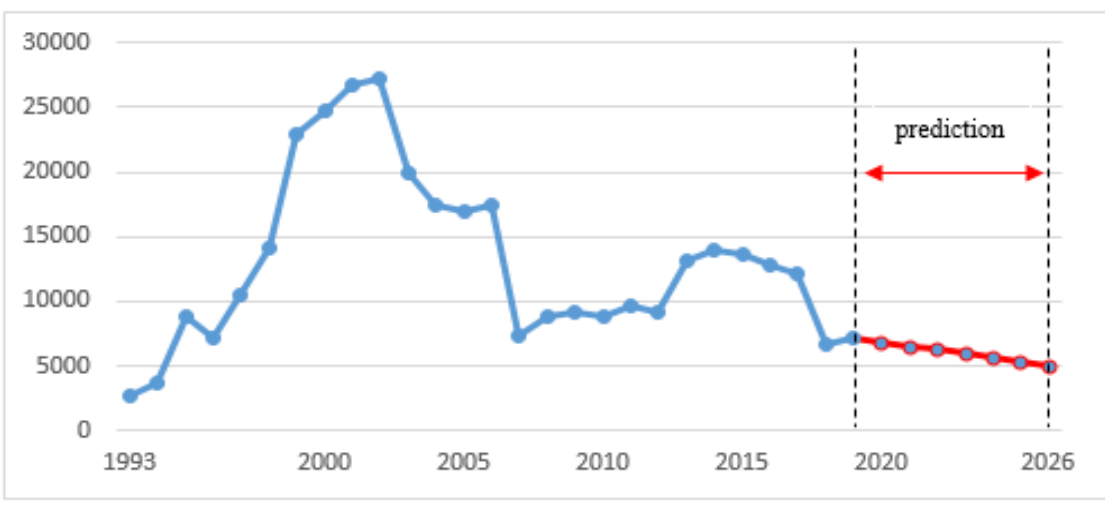

Figure 4. Estimated ISO 9001 Certification Number (Australia)

When the data on South Africa are examined (Table 12, Figure 5), it is seen that the number of ISO 9001 certificates has increased continuously from 1993 to 2000. Although there was a decrease in 2001, the increases generally continued in the following years. The highest number of ISO 9001 certificates was recorded in 2015, 2016 and 2017. In this research, it was predicted that there would be an increase of $22.7 \%$ in the transition from 2019 to 2020 for South Africa, and the number of certificates will increase in the following years. The number of certificates for 2026 is estimated to be 4,626 .

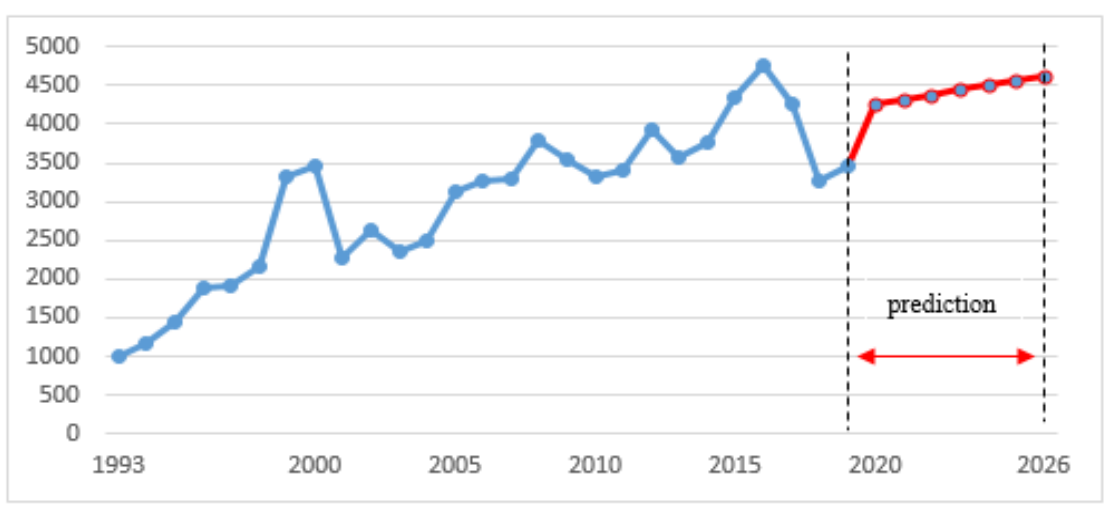

Figure 5. Estimated ISO 9001 Certification Number (South Africa)

In the light of the data in Table 12, it is seen that the number of ISO 9001 certificates in the Russian Federation in the 18 years between 1993 and 2010 (except 2003) has continuously increased. Especially in the transition from 2008 to 2009 , a significant increase of $231.1 \%$ was recorded in the number of certificates. The highest number of ISO 9001 certificates $(62,225)$ was recorded in 2010. After 2010, there has been a significant decrease in the number of certificates (Figure 6). In the period from 2011 to 2019, there was a severe decrease in the number of certificates. The decrease of $78.6 \%$ from 2010 to 2011 is also remarkable. This research predicted that Australia's future ISO 9001 certification number would be in a continuous decrease trend. The number of certificates in 2026 is estimated to be 1,540, with a decrease of $62.7 \%$ compared to 2019 .

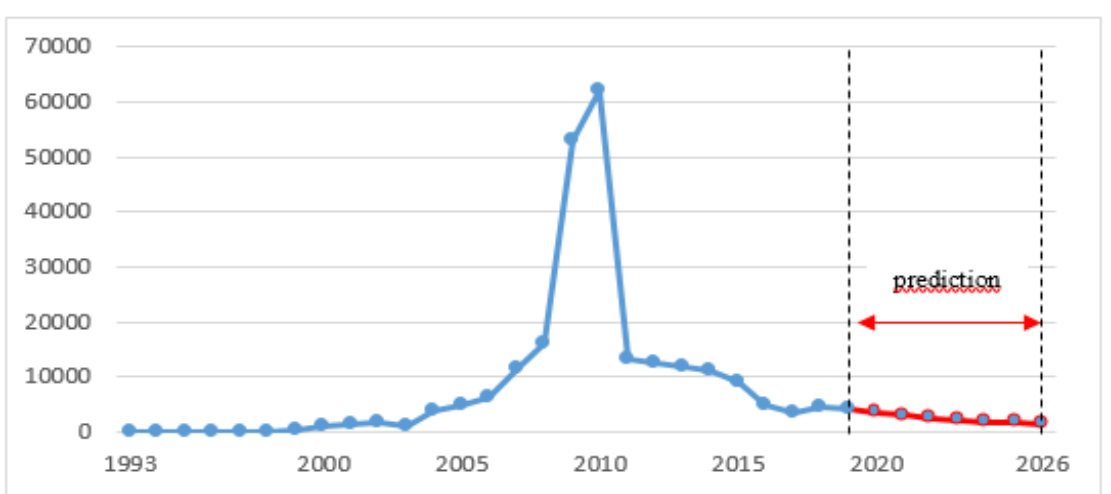

Figure 6. Estimated ISO 9001 Certification Number (Russian Federation)

When the data on Saudi Arabia are examined (Table 12, Figure 7), in the 23 years between 1993 and 2015, it is seen that the number of ISO 9001 certificates (except 2002 and 2003) has continuously increased. The highest number of certificates was reached in 2015. There was a decrease in the number of certificates in 2016, 2017 and 2018. It is predicted in this research that there will be an increase in the 
number of ISO 9001 certificates in the following years in parallel with the increase in 2019. It is estimated that the number of certificates will reach 2.785, with an increase of $26.25 \%$ in 2026 compared to 2019 .

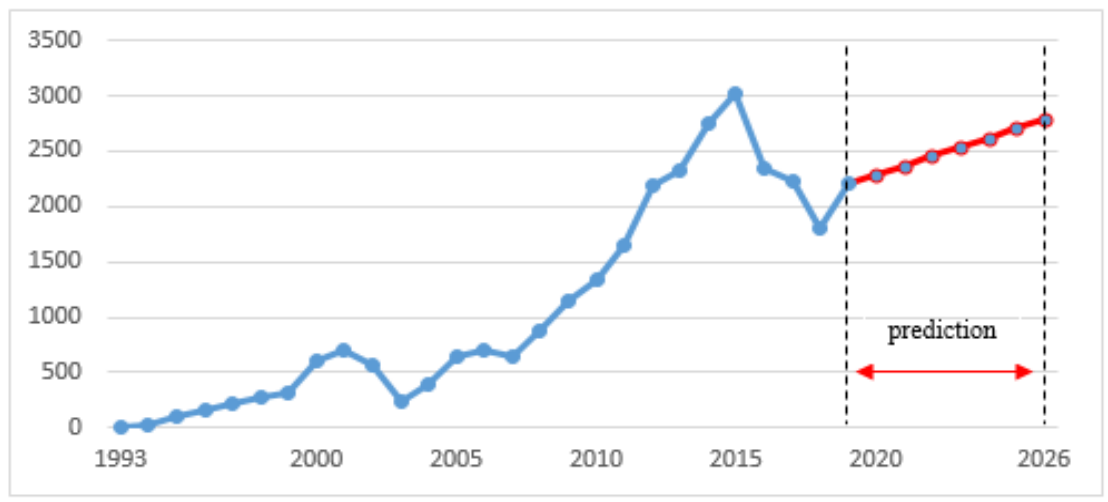

Figure 7. Estimated ISO 9001 Certification Number (Saudi Arabia)

When the numbers of ISO 9001 certificate of Brazil are examined (Table 12), it is seen that the number of certificates continuously increased from 1993 to 2001. Significant increases occurred, especially in 1997, 1998, 1999, 2001, 2007 and 2010. The highest number of certificates was recorded in 2010, 2011 and 2012. After 2011, the number of certificates decreased until 2016. In the period from 2016 to 2019, the number of certificates followed a fluctuating course. This research predicted that the number of certificates would increase continuously for Brazil between 2020 and 2026 (Figure 8), and the number of certificates for 2026 is estimated to be 22,744 . This result is lower than the 2011 value $(28,325)$ when the highest number of certificates is registered.

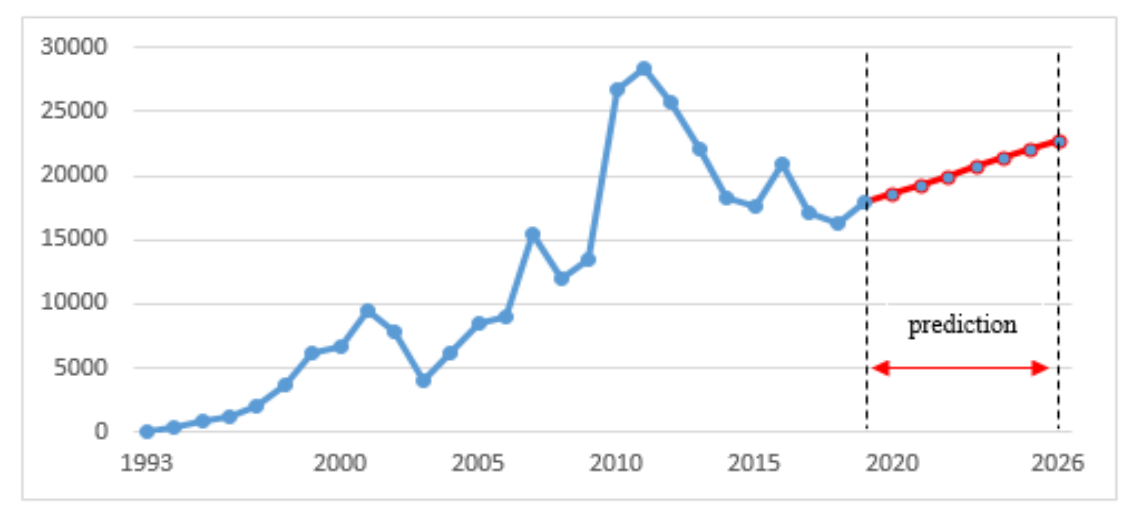

Figure 8. Estimated ISO 9001 Certification Number (Brazil)

In light of the data in Table 12, it is seen that the number of ISO 9001 certificates in Canada continuously increased from 1993 to 2002. When Figure 9 is analysed, there has been a periodic decrease and increase in the number of certificates after 2002. What is striking here is that the increase experienced after the decrease exceeds the previous rising year. The highest number of ISO 9001 certificates $(12,503)$ was reached in 2005. Despite the increases and decreases observed in different periods, in this study, it is predicted that there will be a continuous increase in the number of certificates in 2020-2026 and the number of certificates is estimated to be 11,814 in 2026.

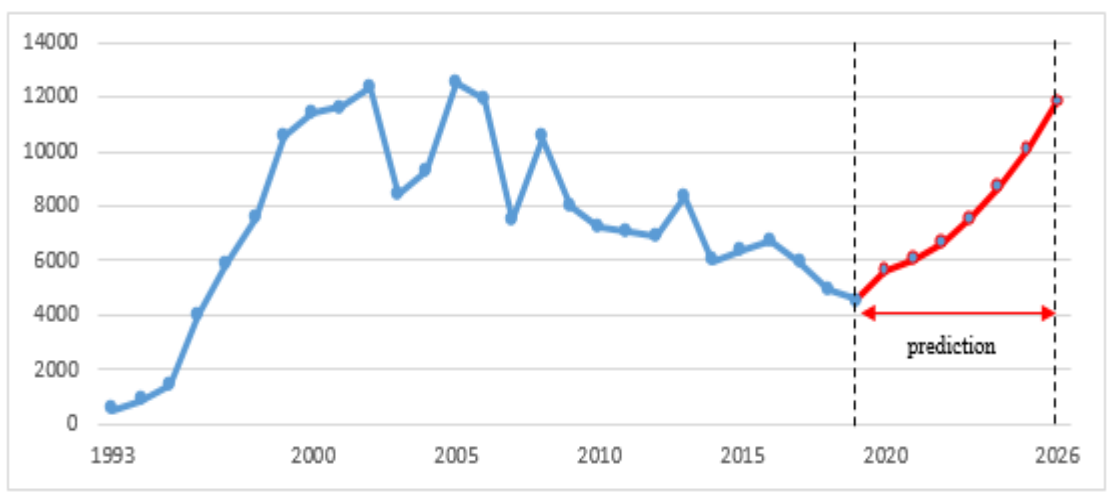

Figure 9. Estimated ISO 9001 Certification Number (Canada) 
When the numbers of ISO 9001 issued to the USA are examined (Table 12, Figure 10), a significant increase is observed in 1995, 1996, 1997, 1998, 1999. In the four years following 2006, there was a continuous decrease. However, the number of certificates increased again in the transition from 2011 to 2013. The number of certificates has decreased continuously since 2015. It is predicted that the number of certificates in the USA will increase continuously. Accordingly, the estimated number of certificates in 2026 was estimated as 37,235, with an increase of $77.7 \%$ compared to 2019 . This result is lower than the 2006 value $(44,883)$ when the highest number of certificates is observed.

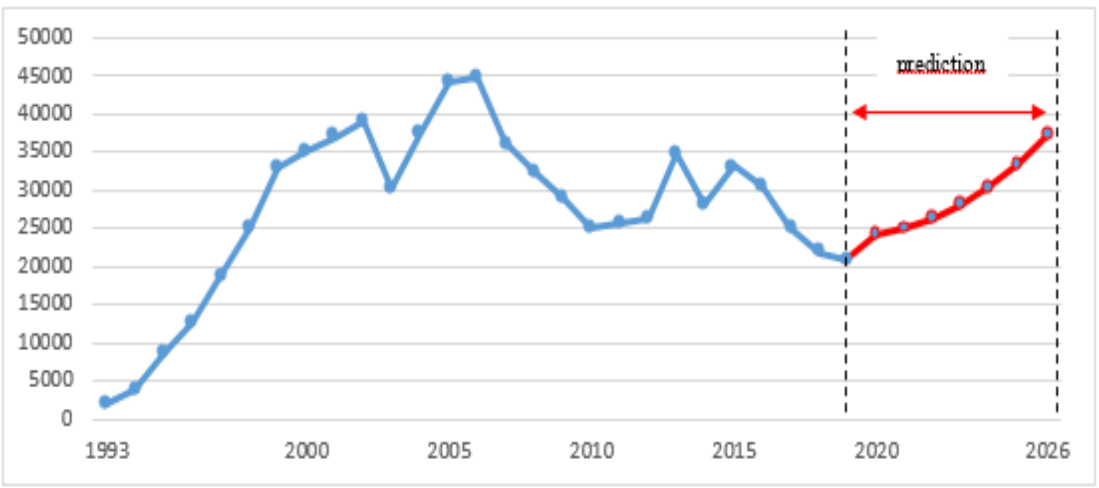

Figure 10. Estimated ISO 9001 Certification Number (USA)

\section{Discussion and conclusion}

When all data (Table 12, Figure 1-10) are evaluated together, especially in the first years (1993-1994) when the statistics were recorded, it is observed that the numbers of certificates in Australia, the USA and South Africa (2,695, 2,059 and 1,007, respectively) are relatively high compared to other countries. In the following years, there was a significant increase in the number of Australia, USA, and India certificates compared to other countries. It is believed that the first standard implementation took place in the European Union countries, especially the United Kingdom (BS 5750 series developed in 1979 by the British Standards Institution) and diffused to the world (Viadiu et al., 2006; Sampaio et al., 2009a; Ikram et al., 2020a). These four countries have close historical relations with the United Kingdom, and economic-political-cultural interactions continue. This may have affected the differentiation of the first statistics for three countries from other countries.

It can be said that the ISO development in Mexico, South Africa and Saudi Arabia has shown more stable growth compared to other countries. Significant increases and decreases have been observed in other countries for many years. Countries are being a part of the global system in today's world, making them more vulnerable to internal and external political and economic uncertainties (Grauwe, 2011; Kelly, Pástor and Veronesi, 2016; Altamirano, 2019; Jiang, Zhu, Tian and Nie, 2019). Besides, it leads countries to develop more protectionist policies (Kee, Neagu and Nicita, 2013; Frieden, 2018; Williams, 2019; Mullan, 2020). The COVID-19 pandemic we have experienced currently and the US-China trade wars are suitable examples of that fact. The economy has been reshaped in recent years, depending on every field's developments, especially technology (Salgado et al., 2016). All these processes more can affect the perception of standard for countries. It can explain the positive and negative trends in ISO 9001 certification statistics.

By 2019, the countries to have the highest number of certificates are India (34,397), USA (20,956), Brazil $(17,952)$, Mexico $(7,741)$, Australia $(7,184)$, Argentina $(6,611)$, Canada $(4,557)$, Russian Federation $(4,134)$, South Africa $(3,464)$, and Saudi Arabia $(2,206)$. In this study, the future certificate numbers of these countries were estimated, and it was predicted that there would be an increase in the number of certificates of all countries except Australia and the Russian Federation. According to 2026 data, it is estimated that Canada $(185.7 \%)$ will have the highest increase in certificate number. Besides, a decrease of about $30.0 \%$ and $62.7 \%$ in the number of certificates is surprisingly envisaged for Australia and the Russian Federation, respectively. Some researchers also predicted that Australia would be down in the future ranking of ISO 9001 certification (Saraiva and Duarte, 2003; Sampaio, Saraiva and Guimarães Rodrigues, 2009a).

Country rankings by the estimated number of certificates for 2026 are as follows: India (43,947), USA $(37,235)$, Brazil $(22,744)$, Canada $(11,814)$, Mexico $(9,977)$, Argentina $(8,433)$, Australia $(5,072)$, South Africa (4,626), Saudi Arabia (2,785) and Russian Federation (1,540). According to the 2019 ranking, South Africa/Saudi Arabia moved up one rank, and Canada moved up three ranks, while Australia and the 
Russian Federation went down two ranks and Mexico went down one rank. No change was observed in the rank of other countries. There are doubtlessly many factors affecting ISO 9001 implementations in each country (Anderson, Daly and Johnson, 1999; Casadesus, Gime and Heras, 2001; Llopis and Tari, 2003; Sampaio, Saraiva and Rodrigues, 2009b; Fonseca and Domingues, 2018; Ikram, Sroufe, Rehman, Shah and Mahmoudi, 2020b). It is generally stated in the literature that the number of businesses will increase in countries due to the economic development, and it will have a positive impact on the ISO 9001 implementation level as well the number of certificates (Saraiva and Duarte, 2003; Sampaio et al., 2009a, 2009c; Clougherty and Grajek, 2014; Manders, 2015; Rodriguez-Arnaldo and Martínez-Lorente, 2020). Within this scope, regarding the 2019 GDP data, countries are ranked as USA (21.4 trillion dollars), India (2.9 trillion dollars), Brazil (1.8 trillion dollars), Canada (1.7 trillion dollars), Russian Federation (1.7 trillion dollars), Australia (1.4 trillion dollars), Mexico (1.25 trillion dollars), Saudi Arabia (793 billion dollars), Argentina (450 billion dollars), and South Africa (351 billion dollars) (W.B., 2019). The University of Minho, University of Coimbra, and National Observatory of Human Resources of Portugal began publishing the World State of Quality (WSQ) index first in 2016 and last in 2018. In the last index, 113 countries were evaluated in 10 different areas, namely Organizations (ISO 9001 Certified Organisations), Professionals, Research, Education, Health, Competitiveness, Social Cohesion, Sustainability, Innovation and Satisfaction, and countries were ranked by comparing their quality performances. According to this index, the maximum quality level is zero, and the lower the score of the index, the higher the country's higher quality level. According to the report published in 2018, the countries in the focus of this study and the index scores were Australia (23.520), Canada (26.891), USA (28.219), Russian Federation (48.853), Mexico (49.471), Argentina (50.558), Brazil (55.676), South Africa (65.342), and India (68.238), respectively. Saudi Arabia was not included in the report (WSQ, 2019). Under normal circumstances, countries with a higher number of certificates are expected to be higher in this ranking. It is understood that the findings do not fully comply with the literature when the rankings of countries based on both GDP and WSQ data are compared with the rankings based on the number of certificates of countries in the focus of this study. This situation points out that the studies to be carried out for the ISO 9001 certificates of the countries should be considered multi-dimensionally.

When the data in Table 12 are examined, it is seen that each country has reached the maximum level number of certificates in different years. The highest number of certificates reached in India in 2007, in Mexico in 2019, in Australia in 2002, in the USA in 2006, in the Russian Federation in 2010 and in Brazil in 2011. The estimated number of countries' certificates (excluding Mexico) for 2026 cannot exceed the highest values recorded in the previous years. This is compatible with the literature proving that the diffusion of ISO 9001 certification may differ from country to country (del Mar Alonso-Almeida, Marimon and Bernardo, 2013; Cabecinhas et al., 2019). Therefore, ISO 9001 will lose its attractiveness for non-certified businesses after reaching a certain level of saturation (Franceschini et al. 2004; Ikram et al., 2020a).

Though it is not one of the focal points of this research, one of the issues that must be discussed most significantly is the effect of ISO 9001 revisions on ISO 9001 statistics. First, we could not find a comprehensive study examining the impact of revisions on ISO 9001 certification diffusion and statistics. This may occur because the literature on ISO 9001 is quite rich. ISO is a global organisation that includes many stakeholder opinions operating in different fields. Therefore, published standards and revisions are prepared with a perspective for both today and the future. The risk analysis approach included in the standard with the 2015 revision can be an example at that point. ISO 9001 standard revisions were carried out in 2000, 2008 and 2015. Businesses are also expected to transit to the new standard approximately in three years. For instance, the number of certificates worldwide in 2003 and 2011 decreased by $11.37 \%$ and $6.19 \%$, respectively, compared to the previous year. The critical point here is a significant increase $(11.57 \%$ ) was observed in the number of certificates in 2018, when the transition period ended (Table 1). Interestingly, the number of ISO 9001 certificates showed an increase following the year when the revision ended (2004, 2012 and 2019).

The data analysis in Table 12 reveals that a significant decrease occurred in the number of certificates for all countries (excluding India and the Russian Federation, respectively) in 2003 and 2018. The same situation was observed in some countries after the revision in 2008 in the following three years. Similarly, after the year in which the revision ended, the number of certificates has generally increased. Some researchers noted that a significant change occurred in the number of certificates during the transition to standard revision (Saraiva and Duarte, 2003; Salgado et al., 2016), but any comprehensive studies could not be reached. We think that the developments in the number of certificates after the ISO 9001 revisions are not coincidental within the light of available data, and the revisions will positively affect the diffusion and number of certificates. It is because revisions will keep the companies' interest in ISO 9001 alive. 
Furthermore, for new businesses in countries that have reached a certain saturation, revisions can be a factor that increases the certificate's attractiveness. ISO (2014) states that ISO 9001 certification statistics, which have been going on for more than two decades, have set a plateau after a significant increase. They predict that certification will follow a positive course both in countries with an established certification tradition and in other countries with market and 2015 revision.

Many studies have been carried out on other MSSs, primarily ISO 14001, with ISO 9001. It has been stated in these studies that the standards generally have common features in many aspects and that one standard facilitates the implementation of the others (Corbett and Kirsch, 2001; Mendel, 2001; Pan, 2003; Viadiu et al., 2006; Casadesus, Marimon and Heras, 2008; Marimon, Casadesús and Heras, 2010; Silva et al., 2016; Marimon and Casadesús, 2017; Heras-Saizarbitoria, Boiral and Allur, 2018; Ferreira, Poltronieri and Gerolamo, 2019; Lira, Salgado and Beijo, 2019; Purwanto, Asbari and Santoso, 2020). The limited number of studies that examine the effect of other MSSs published by ISO on ISO 9001 statistics has drawn our attention. The ISO revisions aim to facilitate integration by eliminating the differences between standards. Thus, companies do not have difficulty in applying different standards. This situation may contribute to companies having more than one standard. On the contrary, an enterprise with a sectoral standard might be indifferent to ISO 9001 due to the similarity between the standards. Thanks to the revisions that facilitate the integration between the standards, the standard diversity of the enterprises will increase, and this will positively affect the number of ISO 9001 certificates, including the countries that reach a certain saturation.

In this study, the past and current ISO 9001 statistics (1993-2019) of the USA, Canada, India, Mexico, Argentina, Australia, South Africa, Saudi Arabia, Brazil and Russian Federation were discussed, and the number of certificates for 2020-2026 was estimated using time series analysis methods. Therefore, the research will primarily contribute to comprehending the ISO 9001 performance and future expectations of these ten G20 countries. Regarding the standards' economic value, the research results will aid certification bodies and consultancy companies, standard practitioners, and other stakeholders, especially ISO, to understand ISO 9001 development trends and learn how the certification market will develop shortly. The research also tried to respond to the literature emphasising the need for more statistical research on the future of ISO 9001. Time series analysis methods can predict the future development of the ISO 9001 standards and others.

Three different time series analysis methods and many different models are used in this study to estimate the future number of ISO 9001 certificates of countries. The models used in this study are the best to explain the data of these ten countries. Therefore, in future predictions, it is impossible to use the models selected in this research paper to estimate the number of certificates for another country. We believe that further research, especially examining the effect of standard revisions and other standards on ISO 9001 diffusion and development are necessary.

\section{Peer-review:}

Externally peer-reviewed

\section{Conflict of interests:}

The author(s) has (have) no conflict of interest to declare.

\section{Grant Support:}

The authors declared that this study has received no financial support

\section{References}

Altamirano, M. (2019). Economic vulnerability and partisanship in Latin America. Latin American Politics and Society, 61(3), 80-103.

Anderson, S. W., Daly, J. D., \& Johnson, M. F. (1999). Why firms seek ISO 9000 certification: regulatory compliance or competitive advantage?. Production and Operations Management, 8(1), 28-43. 
Barros, M. V., Ferreira, M. B., do Prado, G. F., Piekarski, C. M., \& Picinin, C. T. (2020). The interaction between knowledge management and technology transfer: a current literature review between 2013 and 2018. The Journal of Technology Transfer, 45, 1585-1606.

Başaran, B. (2016). The effect of ISO quality management system standards on industrial property rights in Turkey. World Patent Information, 45, 33-46.

Başaran, B. (2018). Integrated management systems and sustainable development. In L. Kounis (Ed.), Quality Management Systems-a Selective Presentation of Case-Studies Showcasing Its Evolution (pp. 1-19). InTechopen, London. ISBN: 978-953-51-3920-1

Cabecinhas, M., Domingues, P., Sampaio, P., \& Arezes, P. (2019). Revisiting diffusion models: Portuguese integrated management systems evolution. In P. M. Arezes, J. S. Baptista, M. P. Barroso, P. Carneiro, P. Cordeiro, N. Costa, R. B. Melo, A. Sérgio Miguel, \& G. Perestrelo (Eds.), Occupational and Environmental Safety and Health (pp. 661-675). Springer, Cham. ISBN: 978-3-030-14729-7

Casadesus, M., Gime, G., \& Heras, I. (2001). Benefits of ISO 9000 implementation in Spanish industry. European Business Review, 13,(6), 327-336.

Casadesus, M., Marimon, F., \& Heras, I. (2008). ISO 14001 diffusion after the success of the ISO 9001 model. Journal of Cleaner Production, 16(16), 1741-1754.

Clougherty, J. A., \& Grajek, M. (2014). International standards and international trade: Empirical evidence from ISO 9000 diffusion. International Journal of Industrial Organization, 36, 70-82.

Corbett, C. J., \& Kirsch, D. A. (2001). International diffusion of ISO 14000 certification. Production and Operations Management, 10(3), 327-342.

del Mar Alonso-Almeida, M., Marimon, F., \& Bernardo, M. (2013). Diffusion of quality standards in the hospitality sector. International Journal of Operations \& Production Management, 33(5), 504-527.

Ferreira, C. D. S., Poltronieri, C. F., \& Gerolamo, M. C. (2019). ISO 14001: 2015 and ISO 9001: 2015: analyse the relationship between these management systems standards and corporate sustainability. Gestão \& Produção, 26(4), e3906.

Fonseca, L. M., \& Domingues, J. P. (2018). Empirical research of the ISO 9001: 2015 transition process in Portugal: Motivations, benefits, and success factors. Quality Innovation Prosperity, 22(2), 16-45.

Franceschini, F., Galetto, M., \& Gianni, G. (2004). A new forecasting model for the diffusion of ISO 9000 standard certifications in European countries. International journal of Quality E reliability management, 21(1), 32-50.

Frieden, J. (2019). The backlash against globalisation and the future of the international economic order. In P. Diamond (Ed.), The Crisis of Globalisation: Democracy, Capitalism, and Inequality in the Twenty-First Century (pp: 43-52). I.B.Tauris, London. ISBN: 978-1-78831-515-9

Grauwe, P. D. (2011). Managing a fragile Eurozone. In CESifo Forum, 12(2), 40-45. München: ifo InstitutLeibniz-Institut für Wirtschaftsforschung an der Universität München. Available at: https:/ / www.econstor.eu/bitstream/10419/166435/1/cesifo-forum-v12-y2011-i2-p040-045.pdf, (Erişim Tarihi: 25.12.2020).

Heras-Saizarbitoria, I., Boiral, O., \& Allur, E. (2018). Three decades of dissemination of ISO 9001 and two of ISO 14001: looking back and ahead. In ISO 9001, ISO 14001, and New Management Standards (pp. 1-15). Springer, Cham. ISBN: 978-3-319-65674-8

Ikram, M., Zhang, Q., \& Sroufe, R. (2020a). Future of quality management system (ISO 9001) certification: novel grey forecasting approach. Total Quality Management E Business Excellence, 1-28.

Do quality, environmental, and social (QES) certifications improve international trade? A comparative grey relation analysis of developing vs. developed countries. Physica A: Statistical Mechanics and its Applications, 545, 123486.

Ikram, M., Sroufe, R., Rehman, E., Shah, S. Z. A., \& Mahmoudi, A. (2020b). Do quality, environmental, and social (QES) certifications improve international trade? A comparative grey relation analysis of developing vs. developed countries. Physica A: Statistical Mechanics and its Applications, 545, 123486.

ISO. (2014). The ISO survey of management system standard certifications - 2014; Executive summary. Available at: https://www.iso.org/files/live/sites/isoorg/files/archive/pdf/en/iso_survey_executivesummary.pdf, (Erişim Tarihi: 25.12.2020). 
ISO. (2015). Quality management systems - Requirements. Fifth edition, ISO.

ISO. (2020a). About Us. Available at: https://www.iso.org/about-us.html, (Erişim Tarihi:19.12.2020).

ISO. (2020b). Members. Available at: https://www.iso.org/members.html?m=MC, (Erişim Tarihi: 21.12.2020).

ISO. (2020c). Management System Standards List. Available at: https://www.iso.org/managementsystem-standards-list.html, (Erişim Tarihi: 11.12.2020).

ISO (2020d). ISO Survey Data. Available https:/ / isotc.iso.org/livelink/livelink?func=11\&objId=18808772\&objAction=browse\&viewType=1, (Erişim Tarihi: 20.12.2020).

Jiang, Y., Zhu, Z., Tian, G., \& Nie, H. (2019). Determinants of within and cross-country economic policy uncertainty spillovers: Evidence from U.S. and China. Finance Research Letters, 31, 195-206.

Kee, H. L., Neagu, C., \& Nicita, A. (2013). Is protectionism on the rise? Assessing national trade policies during the crisis of 2008. Review of Economics and Statistics, 95(1), 342-346.

Kelly, B., Pástor, L'., \& Veronesi, P. (2016). The price of political uncertainty: Theory and evidence from the option market. The Journal of Finance, 71(5), 2417-2480.

Lira, J. M. S., Salgado, E. G., \& Beijo, L. A. (2019). Which factors does the diffusion of ISO 50001 in different regions of the world is influenced?. Journal of Cleaner Production, 226, 759-767.

Llopis, J., \& Tarí, J. J. (2003). The importance of internal aspects in quality improvement. International Journal of Quality E Reliability Management, 20(3), 304-324.

Manders, B. (2015, January 30). Implementation and impact of ISO 9001 (No. EPS-2014-337-LIS). ERIM Ph.D. Series Research in Management. Erasmus Research Institute of Management. Retrieved from http://hdl.handle.net/1765/77412, (Erişim Tarihi: 12.12.2020).

Marimon, F., Casadesús, M., \& Heras, I. (2010). Certification intensity level of the leading nations in ISO 9000 and ISO 14000 standards. International Journal of Quality E Reliability Management, 27(9), 10021020.

Marimon, F., \& Casadesús, M. (2017). Reasons to adopt ISO 50001 energy management system. Sustainability, 9(10), 1740.

Mendel, P. J. (2001), International standardisation and global governance: the spread of quality and environmental management standards. In A. Hoffman, \& M. Ventresca (Eds), Organisations, Policy and The Natural Environment: Institutional and Strategic Perspectives (pp. 407-424). Stanford University Press, Stanford, CA. ISBN: 0-8047-4195-6

Mert, M., \& Çağlar, E. A. (2019). Time series regressions (Chapter 2), Exponential smooting (Chapter 3). Eviews ve Gauss Uygulamalı Zaman Seri Analizleri (pp.41-82). Detay Yayınc1lı, Ankara. ISBN: 978605-254-126-5

Montgomery, D. C., Jennings, C. L., \& Kulahci, M. (2015). Regression analysis and forecasting (Chapter 3), Exponential smooting methods (Chapter 4). Introduction to Time Series Analysis and Forecasting (pp.107-326). John Wiley \& Sons.

Mullan, P. (2020). The rise and rise of protectionism. In Beyond Confrontation: Globalists, Nationalists and Their Discontents (pp. 115-128). Emerald Publishing Limited. ISBN: 978-1-83982-563-7

Nunhes, T. V., Barbosa, L. C. F. M., \& de Oliveira, O. J. (2017). Identification and analysis of the elements and functions integrable in integrated management systems. Journal of Cleaner Production, 142, 32253235.

Pan, J. N. (2003). A comparative study on motivation for and experience with ISO 9000 and ISO 14000 certification among Far Eastern countries. Industrial Management E Data Systems, 103(8), 564-578.

Petricevic, O., \& Teece, D. J. (2019). The structural reshaping of globalisation: Implications for strategic sectors, profiting from innovation, and the multinational enterprise. Journal of International Business Studies, 50(9), 1487-1512.

Prabhakar, A. C., \& Erokhin, V. (2020). Globalisation: reshaping the world economy in the 21st century. In A. C. Prabhakar, G. Kaur, \& V. Erokhin (Eds), Regional Trade and Development Strategies in the Era of Globalisation (pp. 1-24). IGI Global. 
Priede, J. (2012). Implementation of quality management system ISO 9001 in the world and its strategic necessity. Procedia-Social and Behavioral Sciences, 58, 1466-1475.

Purwanto, A., Asbari, M., \& Santoso, P. B. (2020). Effect of integrated management system of ISO 9001: 2015 and ISO 22000: 2018 implementation to packaging industries quality performance at Banten Indonesia. Jurnal Ilmiah MEA (Manajemen, Ekonomi, \& Akuntansi), 4(1), 17-29.

Rodriguez-Arnaldo, O., \& Martínez-Lorente, A. R. (2020). What determinants influence the diffusion of ISO 9001 by countries?. The TQM Journal, ahead-of-print.

Ross, R. S. (2020). It's not a cold war: competition and cooperation in U.S.-China relations. China International Strategy Review, 2(1), 63-72.

Salgado, E. G., Beijo, L. A., Sampaio, P., Mello, C. H. P., \& Saraiva, P. (2016). ISO 9001 certification in the American Continent: a statistical analysis and modelling. International Journal of Production Research, 54(18), 5416-5433.

Sampaio, P., Saraiva, P., \& Guimarães Rodrigues, A. (2009a). An analysis of ISO 9000 data in the world and the European Union. Total Quality Management, 20(12), 1303-1320.

Sampaio, P., Saraiva, P., \& Rodrigues, A. G. (2009b). ISO 9001 certification research: questions, answers and approaches. International Journal of Quality E Reliability Management, 26(1), 38-58.

Sampaio, P., Saraiva, P., \& Rodrigues, A. G. (2009c). A statistical analysis of ISO 9000-related data for European Union ultra-peripheral and Portuguese regions. Quality Management Journal, 16(2), 44-58.

Sampaio, P., Saraiva, P., \& Rodrigues, A. G. (2011). ISO 9001 certification forecasting models. International Journal of Quality E Reliability Management, 28(1), 5-26.

Saraiva, P. M., \& Duarte, B. (2003). ISO 9000: some statistical results for a worldwide phenomenon. Total Quality Management \& Business Excellence, 14(10), 1169-1178.

Silva, M. M., Fonseca, L. M., \& Sousa, S. D. (2016). The impact of ISO 9001: 2015 on ISO 22000 and food safety management systems (FSMS). Calitatea, 17(152), 81-85.

Simon, A., Yaya, L. H. P., Karapetrovic, S., \& Casadesús, M. (2014). An empirical analysis of the integration of internal and external management system audits. Journal of Cleaner Production, 66, 499506.

Viadiu, F. M., Fa, M. C., \& Saizarbitoria, I. H. (2006). ISO 9000 and ISO 14000 standards: an international diffusion model. International Journal of Operations E Production Management, 26(2), 141-165.

Williams, N. (2019). The resilience of protectionism in U.S. trade policy, Boston University Law Review, 99, 683 - 718. Available at: https://www.bu.edu/bulawreview/files/2019/03/WILLIAMS.pdf, (Erişim Tarihi: 21.12.2020).

World Bank (WB). (2019). GDP Data. Available https://data.worldbank.org/indicator/NY.GDP.MKTP.CD, (Erişim Tarihi: 15.12.2020).

World State of Quality (WSQ). (2019). 2018 world quality scoreboard, Available at: http://wsq.dps.uminho.pt/wsq_report2018/wsq_report2018.pdf, (Erişim Tarihi: 15.12.2020). 